\title{
Segmental Dynamics of Atactic Polypropylene As Revealed by Molecular Simulations and Quasielastic Neutron Scattering
}

\section{Oscar Ahumada, ${ }^{\dagger}$ Doros N. Theodorou,,,,$+ \neq$ Alessandro Triolo, $\$$ Valeria Arrighi, ${ }^{\S}$ Costas Karatasos," and J ean-Paul Ryckaert"}

Department of Chemical Engineering, University of Patras and ICE/ HT-FORTH, GR-26500 Patras, Greece, Chemistry Department, Heriot-Watt University

E dinburgh, EH14 4AS, United Kingdom, and Laboratoire de Physique des Polymères, CP223, Université Libre de Bruxelles, Boulevard de Triomphe, B-1050 Bruxelles, Belgique

Received October 18, 2001; Revised Manuscript Received May 1, 2002

\begin{abstract}
The dynamics of atactic polypropylene has been explored with quasielastic neutron scattering (QENS) measurements in the temperature range $4-460 \mathrm{~K}$ and momentum transfer range $\mathrm{q}=0.2-2.25$ $\AA^{-1}$. In parallel, molecular dynamics simulations of the same polymer have been conducted in the temperature range $260-600 \mathrm{~K}$, using both a fully atomistic model and a model with united-atom methyl groups. In conjunction with the second model, a computational procedure for introducing the motion of methyl hydrogens a posteriori is proposed and tested against the fully atomistic simulation results. Simulated intermediate incoherent scattering functions I (q,t) reveal an initial exponentially decaying regime of duration ca. 1 ps, which is dominated by bond angle bending vibrations and torsional oscillations, as well as features attributable to torsional transitions of the methyl groups and to correlated conformational transitions of the backbone bonds at longer times. The time decay of I (q,t) beyond 1 ps is well-described by stretched exponential functions, the stretching exponent being around 0.5 at $600 \mathrm{~K}$ and decreasing with decreasing temperature. Analysis of the atomistic simulation trajectories yields distributions of relaxation times with a distinct log-Gaussian peak characteristic of methyl motion, from which a Gaussian distribution of activation energies for methyl torsional transitions with mean around $15 \mathrm{~kJ} / \mathrm{mol}$ and standard deviation around $3 \mathrm{~kJ} / \mathrm{mol}$ is extracted, in excellent agreement with QENS estimates. Torsional transitions of different methyls occur essentially independently of each other. QENS experiments reveal a nondecaying elastic contribution to the scattering over the time window of the measurement, which is not seen in the simulations. Apart from this, computed I $(q, t)$ and incoherent dynamic structure factor $S(q, \omega)$ curves are in very favorable agreement with the measured QE NS spectra and with earlier NMR data on atactic polypropylene.
\end{abstract}

\section{Introduction}

Dynamics in polymer systems involve motion that is spread over a broad range of time scales, 1,2 from the very slow processes involved in viscous flow to fast dynamical processes detected in the subpi cosecond range. Among these motions, most frequently investigated are the segmental dynamics or $\alpha$-relaxation, which is connected with the glass transition phenomena characteristic of any glass-forming system, and the secondary relaxations, which are believed to be responsible for shaping the mechanical properties of glassy polymers. However, in polymers having side groups, other processes related to very local internal motions are al so expected. In fact, at low temperatures, where the polymer chain backbones are more or less frozen and secondary relaxations are very slow, motions associated with small units remain active. This is the case for atactic polypropylene (aPP), where the side group is a methyl unit.

In the present paper, the dynamics of aPP has been studied using molecular simulations and quasielastic

* To whom correspondence should be addressed at the National Technical University of Athens. Telephone: +3010-772-

3157. Telefax: +3010-772-3184. E-mail: doros@central.ntua.gr. + University of Patras and ICE/HT-FORTH

‡ Present address: Department of Materials Science and Engineering, School of Chemical Engineering, National Technical University of Athens, 9 Heroon Polytechniou Street, Zografou Campus, GR-157 80, Athens, Greece.

$\S$ Heriot-Watt University.

" Université Libre de Bruxelles. neutron scattering (QENS) measurements. The positions of all hydrogens of the polymer are tracked in the course of MD simulation trajectories in a manner that allows calculating observables of QENS experiments, which are then compared directly against the measurements. Six types of motion are tracked by the simulation: fast torsional librations of the methyls within their energy wells, torsional transitions of the methyls between different energy wells, bond angle bending vibrations of skeletal and pendant bonds, torsional librations around skeletal bonds along the chains, conformational transitions of the skeletal bonds, and translational displacement of entire chains. All these motions are followed naturally in the course of molecular dynamics (MD) simulations using a detailed molecular model (model I), in which all atoms are represented explicitly. To track longer time motion at modest computational cost, a more coarse-grained molecular model (model II) is also simulated, in which methyl groups are represented as united atoms. This second model was designed to give a satisfactory compromise between real ism and computational tractability; dynamical predictions from it have al ready been compared to NMR relaxation and solid-state NMR measurements. ${ }^{3}$ In model II, the first two types of motion mentioned above (torsional librations and jumps of the methyl groups) have to be built in a posteriori in the simulation trajectories; here this is done using a simple model for methyl motion, which is based on an experimentally derived distribution of torsional energy barriers for the methyls. The latter 
distribution is consistent with MD trajectories generated using the fully atomistic model I.

Over the past 5 years, several attempts have been made to exploit MD trajectories and calculate the coherent and incoherent dynamic structure factors, ${ }^{4-7}$ which are experimentally available from neutron scattering. Although most of the studies published to date deal with polymer melt dynamics, few reports have appeared in the literature looking at molecular motion bel ow the glass temperature, e.g., methyl group rotation in polyisoprene ( $\mathrm{PI})^{8,9}$ and poly(vinyl methyl ether). ${ }^{10}$

It has become evident that atomistic simulations provide an excellent tool for investigating molecular motion in unentangled polymer melts. Smith et al.4,7 reported a detailed quantitative comparison between MD simulations and the incoherent dynamic structure factor from time-of-flight measurements carried out on a long-chain alkane $\left(\mathrm{n}-\mathrm{C}_{100} \mathrm{H}_{202}\right)$ at $504 \mathrm{~K}$. Detailed analysis of the MD data indicated that the time decay of the incoherent intermediate scattering function I $(q, t)$ in the range $0.1-10$ ps results from a combination of torsional librations and conformational jumps, the latter becoming increasingly important at low q. Neutron spin-echo measurements carried out on the same alkane were also compared to MD simulations, ${ }^{5,7}$ in order to test the validity of the Rouse model.

The first MD determination of the coherent dynamic structure factor was published by Moe et al. ${ }^{6}$ The static, $\mathrm{S}(\mathrm{q})$, and dynamic structure factors were cal culated and compared to measurements carried out by Zorn et al. ${ }^{11}$ on PI melts. The simulations were able to reproduce many important qualitative features of the neutron scattering measurements, but due to differences in microstructure (the simulations used $100 \%$ cis-PI while the experiments were carried out on a 76:18:6 cis:trans: vinyl PI sample) and temperature, it was not possible to achieve a detailed quantitative comparison between experimental data and simulations. It was concluded that the time decay of $I(q, t)$ can be attributed to intramolecular dynamics almost entirely, throughout the q range studied $\left(1.2-3 \AA^{-1}\right)$. In addition to polyethylene and PI, a detailed comparison between experiments and simulations has been reported by Smith et al. ${ }^{7}$ for polybutadiene (PB) melts and by both Mos et al. ${ }^{12}$ and de Leeuw et al. ${ }^{13}$ for poly(ethylene oxide) (PEO)

More recently, MD simulations have been exploited to study the dynamic behavior of more complex systems, such as PEO/salt systems ${ }^{12-14}$ and polymer blends. ${ }^{15} \mathrm{As}$ shown by the agreement between experiments and simulations, MD is highly suited for investigating dynamic changes that take place upon blending. In a miscible blend of cis-1,4-PI (70 wt \%) with 1,2-PB, both MD simulations and QENS measurements by Doxastakis et al. ${ }^{15}$ revealed differences in segmental mobility between the two components, even at temperatures 100 $\mathrm{K}$ above the glass temperature, emphasizing the importance of intramolecular factors. Similarly, simulations can reproduce qualitatively the slowing down effect on PEO relaxation due to salt addition. ${ }^{12,13}$

MD simulations have been valuable in investigating many aspects of dynamics probed by other relaxation measurements than dynamic neutron scattering. Smith et al. ${ }^{16}$ computed ${ }^{13} \mathrm{C}$ nuclear magnetic resonance (NMR) spin-lattice relaxation times $\left(T_{1}\right)$ and nuclear Overhauser enhancement (NOE) values, as well as dielectric relaxation spectra for PEO melts. MD predictions were found to be in good agreement with NMR observables over a wide range of temperatures, as well as with the dielectric relaxation strength and maximum dielectric loss frequency. Relaxation times extracted from the time autocorrelation functions of $\mathrm{C}-\mathrm{H}$ bonds, torsion angles, and molecular dipole moments were found to exhibit a non-Arrhenius temperature dependence. Detailed comparisons of the local chain dynamics of 1,4-PB melts, as determined from experiments and MD simulations at $353 \mathrm{~K}$, were presented by Smith et al. ${ }^{17}$ The local dynamics was measured through ${ }^{13} \mathrm{C} N M R \mathrm{~T}_{1}$ and NOE values and through neutron spin-echo (NSE) spectroscopy. The predicted dynamics was found to be too fast, necessitating a revision of the molecular model, after which predicted properties were within $20-30 \%$ of experiment. Recently, ${ }^{18}$ this PB work was extended to cover the temperature range $273-400 \mathrm{~K}$.

Comparisons between simulated and measured dynamics have al so been performed for polypropylene (PP). Destrée et al. ${ }^{19}$ studied local dynamics in solution by ${ }^{13} \mathrm{C}$ relaxation and by MD. Both experiments and simulations suggested a slightly higher mobility of meso sequences in comparison with racemic sequences. Antoniadis et al. ${ }^{3}$ performed MD simulations of aPP melts, accumulating time autocorrelation functions for pendant bonds and skeletal torsion angles. Predicted correlation times were in good agreement with existing NMR measurements. Antoniadis et al. ${ }^{20}$ al so studied the effect of tacticity on segmental dynamics in the melt; they found isotactic PP to exhibit significantly faster dynamics than syndiotactic PP at the same temperature, with aPP Iying between. Subsequently to these cal culations, Ediger et al. ${ }^{21,22}$ performed careful ${ }^{13} \mathrm{C}$ NMR relaxation measurements of the local dynamics of PP, and compared with the results of Antoniadis et al. NMR correlation times of aPP were in reasonable agreement with MD values, but the temperature dependences of $\mathrm{T}_{1}$ and NOE values exhibited some differences from those calculated from MD. ${ }^{21}$ Direct NMR measurements on melts of different tacticities 22 confirmed the simulation prediction that the segmental dynamics slows down as one goes from isotactic to atactic to syndiotactic PP, differences in the experiment being generally smaller than those predicted by MD. A refinement of simulation parameters was recommended.

The present paper is largely based on the aPP model of Antoniadis et al. ${ }^{3}$ Refinements in the modeling have been introduced in two directions: (a) consideration of a fully atomistic model, incorporating an explicit representation of the methyls (model I), and (b) a posteriori inclusion of the methyl torsion dynamics in trajectories generated by the model of Antoniadis et al. (model II). The observables of QENS are extracted and compared to QENS measurements, conducted on aPP for the first time. The dynamics, as revealed by QENS, is also compared against earlier NMR measurements and simulation predictions of the NMR observables.

\section{Molecular Simulations with Model I}

Details on the force field parametrization and on the preparation of the initial configurations of model I can be found in ref 23 and will not be repeated here. All the molecular dynamics runs with this model were performed with the DLPROTEIN package. ${ }^{24}$ The simulated system was equilibrated at four temperatures, namely $400,458,500$, and $546 \mathrm{~K}$, and subsequently trajectories 
longer than 1 ns were generated in the constant energy (NVE) ensemble.

\section{Molecular Simulations with Model II}

Model II has been explained in ref 3. In this model, polypropylene chains are represented in terms of explicit skel etal carbon (C) and pendant hydrogen $(\mathrm{H})$ atoms, but methyl groups are lumped into single quasiatomic entities $\left(\mathrm{R}=\mathrm{CH}_{3}\right)$. All bond lengths are kept fixed, while $\mathrm{C}-\mathrm{C}-\mathrm{C}$ and $\mathrm{C}-\mathrm{C}-\mathrm{R}$ bond angles are subject to stiff harmonic potentials. The positions of the pendant hydrogens are determined uniquely by the positions of the heavy sites ( $C$ and $R$ ), and the masses of the pendant hydrogens are collapsed onto the carbon atoms to which they are connected. Nonbonded interactions between $\mathrm{H}$, $C$, and $R$ sites separated by more than two bonds along the same chain, or between sites bel onging to different chains, are described by Lennard-- ones potentials. To represent both the conformational energetics and the cohesive forces of the polymer reasonably realistically, a distinction is made between "Iocal" and "nonlocal" nonbonded interaction potentials. In addition, there is a torsional potential associated with skeletal bonds. The detailed parametrization of model II has been reported in ref 3 and will not be repeated here.

The molecular simulation strategy for tracking the motion of hydrogens with model II consists of two parts: (1) conducting long MD simulations of model II to generate trajectories for the bulk polymer within the united atom representation of the methyl groups and (2) generating separate trajectories for the evolution of the torsion angle of each methyl group with respect to the backbone. The latter trajectories are superposed on the former by "unfolding" the methyls from a unitedatom into an explicit representation in the bulk polymer simulations.

As in ref 3, the model system used in generating the MD trajectories with model II consisted of two parent chains having a degree of polymerization of 76 (molar mass $3208 \mathrm{~g} / \mathrm{mol}$ ) in a cubic cell of initial edge length $23.42 \AA$ with three-dimensional periodic boundary conditions. The sequence of stereochemical configurations of the pseudoasymmetric carbon atoms along each parent chain was generated by assuming a Bernoullian distribution of meso and racemo diads with a probability of occurrence of a meso diad equal to 0.48 , as observed experimentally in equilibrium epimerized aPP. ${ }^{25}$ The MD simulations were carried out in the isothermalisobaric (NPT) ensemble at different temperatures, ranging from 260 to $600 \mathrm{~K}$. Configurations from the production phase of each simulation were recorded every 1 ps during a total time of 1-5 ns, depending on the temperature. In some cases a higher recording frequency was used to analyze the short-time dynamics.

Our reconstruction of the local dynamics of methyls with model II rests on the idea that the torsion angle $\phi$ around the methyl "stem", i.e., around the $\mathrm{C}-\mathrm{CH}_{3}$ bond connecting the methyl to the backbone, is governed by a 3-fold symmetric potential of mean force $\mathrm{V}(\phi)$ of the form

$$
\mathrm{V}(\phi)=\frac{1}{2} \mathrm{E}_{\mathrm{A}}[1-\cos (3 \phi)]
$$

In general, $\mathrm{V}(\phi)$ is affected by both intramolecular and intermolecular interactions, so the value of $\mathrm{E}_{\mathrm{A}}$ is allowed to be different between different methyls in the system.
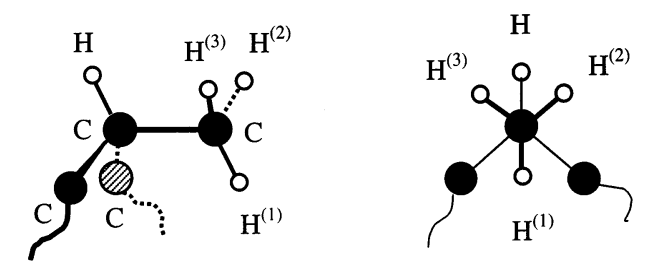

Figure 1. Reconstruction of methyl hydrogens from unitedatom representation of a methyl group. The $\mathrm{C}-\mathrm{C}$ bond connecting the methyl to the backbone ("methyl stem") is a 3 -fold axis of symmetry for the methyl.

The value at $\phi=0$ is taken to correspond to the staggered conformation shown in Figure 1 (the methyl bond $\mathrm{C}-\mathrm{H}^{(1)}$ being coplanar with the methyl stem and with the $\mathrm{C}-\mathrm{H}$ bond of the methine group to which the methyl is connected). For all methyls, $\mathrm{C}-\mathrm{H}$ bonds are assumed to be $1.10 \AA$ long and to form an angle of $110^{\circ}$ with the methyl stem.

A reasonable description of the dynamics of methyl motion around the stem subject to $\mathrm{V}(\phi)$ can, in general, be given by the stochastic differential (Langevin) equation

$$
\mathrm{I} \ddot{\phi}+\xi \dot{\phi}+\frac{\partial \mathrm{V}}{\partial \phi}-\mathrm{T}_{\text {Langevin }}=0
$$

where $\mathrm{I}=5.3 \times 10^{-47} \mathrm{~kg} \mathrm{~m}^{2}$ is the moment of inertia of a methyl group around its stem, $\xi$ is a friction coefficient, and $T_{\text {Langevin is a stochastic, fluctuating torque that is }}$ isotropically distributed in space, loses memory of its prior orientation practically instantaneously, and has a mean square magnitude proportional to the friction coefficient $\zeta$ and to the absolute temperature T, according to the requirements of the fluctuation-dissipation theorem. The quantities $\xi, T_{\text {Langevin, }}$ and $\mathrm{V}(\phi)$ provide a collective description of the effects of the environment on the motion of $\phi$ and arise formally when the equations of motion of the whole system are projected onto the degree of freedom $\phi$. In writing down eq 2, we have already neglected any coupling between different methyls in their rotational motion around their axes; this could be justified on the basis of the relatively large distance between different methyls al ong the backbone of aPP and is confirmed by our fully atomistic simulations with model I (see section 5).

In connection with eqs 1 and 2 , we introduce the foll owing justifiable assumptions:

(1) We assign values to the free energy barrier $E_{A}$ (eq 1) to different methyls in the system from the Gaussian, temperature-independent distribution

$$
g\left(E_{A}\right)=\frac{1}{\sigma \sqrt{2 \pi}} \exp \left[-\frac{\left(E_{A}-E_{o}\right)^{2}}{2 \sigma^{2}}\right]
$$

with $\mathrm{E}_{0}=14.3 \mathrm{~kJ} / \mathrm{mol}$ and $\sigma=3.3 \mathrm{~kJ} / \mathrm{mol}$. These distribution and parameter values are based on our lowtemperature quasielastic neutron scattering measurements. ${ }^{26}$ They are fully supported by our atomistic MD simulations using model $\mathrm{l}$, which allow tracking the methyl dynamics explicitly (see section 5). We note that atomistic calculations of $E_{A}$ distributions in polymer glasses and melts have been presented by Davies and co-workers, ${ }^{27,10}$ and Gaussian distributions have been invoked in earlier experimental works. 28,29

(2) We assume that the friction constant $\xi$ is low enough to justify neglect of the frictional term in eq 2 in describing motion within the methyl free energy 
wells, yet large enough for the dynamics of transitions between wells to be describable by an activated rate expression with transmission coefficient equal to 1 . On the basis of the Kramers analysis, ${ }^{30,31}$ the precise condition for this picture to be applicable is

$$
3 \sqrt{\frac{E_{A}}{2 l}} \frac{k_{B} T}{E_{A}}<\frac{\zeta}{l}<3.6 \sqrt{\frac{E_{A}}{2 l}}
$$

with $E_{A}$ measured in $\mathrm{J} /$ molecule and $k_{B}$ being the Boltzmann constant.

(3) We assume that the barrier $E_{A}$ is high relative to the thermal energy $k_{B} T$. From eq 3 and the values of $\mathrm{E}_{\mathrm{o}}$ and $\sigma$ associated with it, this is a reasonable assumption for the temperature of interest here.

Under the assumptions stated above, the motion of $\phi$ described by eq 2 becomes essentially a harmonic oscillation within the wells defined by eq 1 at $\phi_{0}=0$, $2 \pi / 3$, and $-2 \pi / 3$ with restoring torque $-\left(\partial^{2} \mathrm{~V} / \partial \phi^{2}\right)_{\phi=\phi_{0}}(\phi$ $\left.-\phi_{0}\right)=-9 / 2 \mathrm{E}_{\mathrm{A}}\left(\phi-\phi_{0}\right)$, intercepted by infrequent transitions from a well into an adjacent one. The oscillation within the wells has angular frequency

$$
\omega=3 \sqrt{\frac{E_{A}}{2 l}}
$$

and amplitude

$$
A_{o}=\frac{2}{3} \sqrt{\frac{k_{B} T}{E_{A}}}
$$

The rate constant for well-to-well transitions is given by the simple transition-state theory in the harmonic approximation

$$
\mathrm{k}^{\mathrm{TST}}=\frac{\omega}{2 \pi} \exp \left(-\frac{\mathrm{E}_{\mathrm{A}}}{\mathrm{k}_{\mathrm{B}} \mathrm{T}}\right)
$$

With this simple model, all parameters of the rotational motion of a methyl around its stem are known once the barrier height $\mathrm{E}_{\mathrm{A}}$ is specified. Thus, simulating this motion becomes straightforward: As long as the methyl is executing torsional librations in its well, the torsion angle $\phi$ evol ves according to

$$
\phi=\phi_{\mathrm{o}}+\mathrm{A}_{\mathrm{o}} \sin (\omega \mathrm{t})
$$

where $t$ is the time since its last torsional jump. The time $t$ at which the next torsional jump will occur is picked from an exponential distribution with mean $1 /\left(2 k^{T S T}\right)$, consistent with the Poisson statistics of transition-state theory. Immediately after the jump the torsion angle is placed at $\phi_{0}+2 \pi / 3$ or at $\phi_{0}-2 \pi / 3$ with equal probability, i.e., at the exact minimum of the adjacent well into which the jump occurs. It is likely that the simple, zero-friction harmonic expression, eq 8 , does not capture all the complexities of torsional librations within the wells. Nevertheless, given the small amplitude of these librations relative to the angular displacements effected in the course of torsional jumps, we feel that the error introduced will not bevery significant.

This local dynamics is followed in parallel with the MD trajectory for each methyl group in the system and

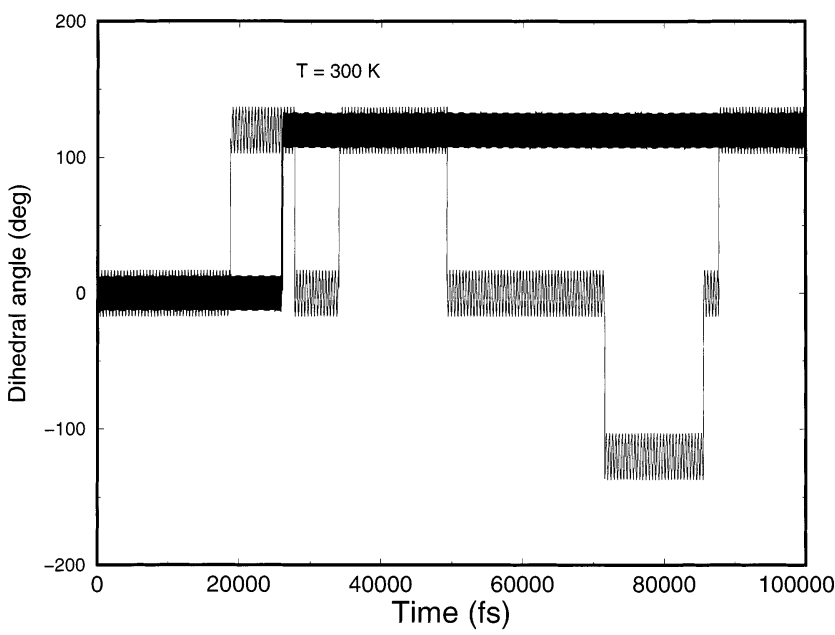

Figure 2. Torsion angle $\phi$ as a function of time for two different methyl groups in the aPP system (model II) at $300 \mathrm{~K}$

superposed on the MD trajectory, so the positions of all hydrogens (both pendant and methyl) in aPP are ultimately known at each MD time step.

At this point, it is useful to mention some characteristic time scales governing the simple model of methyl motion invoked in model II. According to eq 5 , the mean value of $\mathrm{E}_{\mathrm{A}}=14.3 \mathrm{~kJ} / \mathrm{mol}$ corresponds to a torsional libration frequency of the methyls equal to $\omega=4.5 \times$ $10^{13} \mathrm{~s}^{-1}$ or to a librational period of $2 \pi / \omega=0.14 \mathrm{ps}$. On the other hand, the well-to-well transition rate constants $\mathrm{k}^{\mathrm{TST}}$ calculated from eq 7 and the corresponding in-well residence times $\tau_{\text {jump }}=1 /\left(2 \mathrm{k}^{\mathrm{TST}}\right)$ between torsional transitions of a methyl are as follows: $\mathrm{k}^{\mathrm{TST}}=9.6 \times 10^{9}$ $\mathrm{s}^{-1}$ and $\tau_{\text {jump }}=52$ ps at $260 \mathrm{~K} ; \mathrm{k}^{\text {TST }}=5.2 \times 10^{10} \mathrm{~s}^{-1}$ and $\tau_{\text {jump }}=10 \mathrm{ps}$ at $350 \mathrm{~K} ; \mathrm{k}^{\mathrm{TST}}=1.6 \times 10^{11} \mathrm{~s}^{-1}$ and $\tau_{\text {jump }}=3.2 \mathrm{ps}$ at $450 \mathrm{~K} ; \mathrm{k}^{\mathrm{TST}}=3.1 \times 10^{11} \mathrm{~s}^{-1}$ and $\tau_{\text {jump }}$ $=1.6 \mathrm{ps}$ at $550 \mathrm{~K}$; and $\mathrm{k}^{\mathrm{TST}}=4.1 \times 10^{11} \mathrm{~s}^{-1}$ and $\tau_{\mathrm{jump}}=$ $1.2 \mathrm{ps}$ at $600 \mathrm{~K}$.

Figure 2 displays traces of the time evolution of the dihedral angle $\phi$ for two methyl groups at $300 \mathrm{~K}$, as simulated with model II. The dihedral angle was recorded every femtosecond. The small-amplitude, highfrequency torsional oscillations intercepted by infrequent $120^{\circ}$-jumps are evident. The waiting time between jumps is approximately 15 ps for the faster of the two methyls and considerably longer for the slower one, which is characterized by a higher $\mathrm{E}_{\mathrm{A}}$. As expected from eq 6, the methyl with a higher torsional energy barrier exhibits a smaller amplitude during its in-well oscillations.

\section{Experimental Section}

All samples of atactic polypropylene used in the measure ments were obtained from commercial isotactic polypropylene samples through extraction and fractionation with respect to mol ecular weight. The samples measured on the IN 10 instrument at ILL and on the IRIS instrument at ISIS had a number average molar mass of $64000 \mathrm{~g} / \mathrm{mol}$ (as determined by GPC) and a glass temperature of $266 \mathrm{~K}$ (as measured by DSC). The sample measured on the NEAT instrument at BENSC had a number average molar mass of $2900 \mathrm{~g} / \mathrm{mol}$ and a glass temperature of $255 \mathrm{~K}$. Both samples had polydispersities in the range 1.2-1.3.

QE NS measurements were carried out on the IN 10 backscattering spectrometer at the Institut Laue Langevin (ILL), Grenoble, France. ${ }^{32}$ This instrument has a $1 \mu \mathrm{eV}$ resolution $\left(4.1 \times 10^{-9} \mathrm{~s}\right)$ measured as full width at half peak height and an accessible q range from 0.20 to $2.00 \AA^{-1}$. Incoherent el astic 


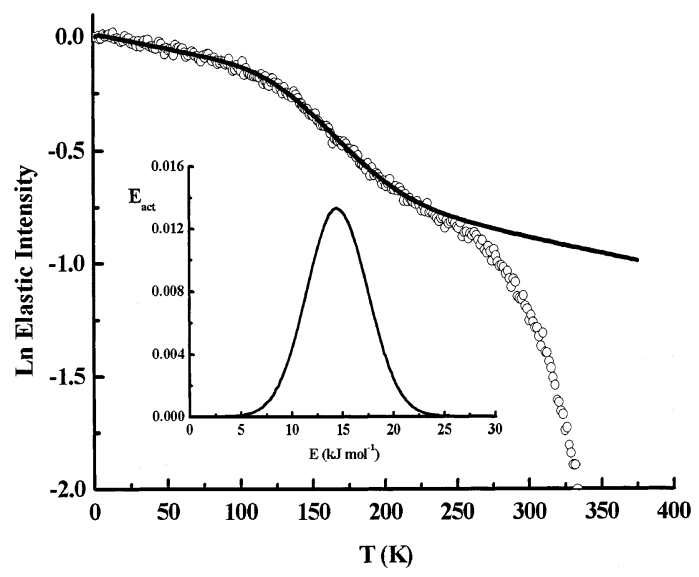

Figure 3. Elastic intensity $S(q, \omega \approx 0)$ as a function of temperature, as obtained from the fixed-window scan measurements collected at IN 10 with $q=1.96 \AA^{-1}$ (points) and its fit, as described in ref 26 (line). The distribution of activation energies $g\left(E_{A}\right)$ extracted from the fit is shown in the inset.

neutron scattering measurements were performed using the fixed-window technique 32,33 in the temperature range $2-400$ $\mathrm{K}$. In this case, the neutron scattered intensity is monitored within a narrow energy range centered at $\omega=0$ (whose width is related to the instrumental resolution), as a function of temperature and scattering vector, q; only those neutrons that are elastically scattered are counted. The reader is reminded that, here and in the following, the meaning of "elastic" is relative to the particular instrumental resolution used. Any process with characteristic time longer than the time corre sponding to the resolution will look as elastic for a given instrument. An extrapolation procedure was used to normalize the data at each q value; the logarithm of the elastic intensity, $\mathrm{S}(\mathrm{q}, \omega \approx 0)$, was plotted versus temperature, and the lowtemperature data were fitted to provide a value of the extrapolated intensity at $\mathrm{T}=0$. The window scan data of atactic polypropylene were used to evaluate the mean activation energy, $E_{0}$, and the width of the distribution of activation energies, $\sigma$, associated with the rotational motion of the methyl groups (see Figure 3). A description of the procedure adopted is reported elsewhere. ${ }^{26}$

In the following sections we will make use of an "apparent" mean squared atomic displacement of hydrogen atoms, $\left\langle r^{2}\right\rangle_{\text {app. }}$. This is obtained from the measured elastic intensity $\mathrm{S}(\mathrm{q}, \omega \approx 0)$ by postulating

$$
\mathrm{S}(\mathrm{q}, \omega \approx 0)=\mathrm{e}^{-\left\langle\mathrm{r}^{2}\right\rangle \mathrm{app} \mathrm{q}^{2} / 6}
$$

or, more precisely,

$$
\frac{\left\langle\mathrm{r}^{2}\right\rangle_{\mathrm{app}}}{6}=-\frac{\partial \ln \mathrm{S}(\mathrm{q}, \omega \approx 0)}{\partial\left(\mathrm{q}^{2}\right)}
$$

Eq 9 is inspired by the picture of harmonic motion within a well. It is emphasized that $\left\langle r^{2}\right\rangle_{\text {app }}$ is only an apparent mean squared displacement. In other words, eq 9 provides a convenient way for expressing the elastic incoherent structure factor (EISF) in units of squared length.

At temperatures below $100 \mathrm{~K},\left\langle\mathrm{r}^{2}\right\rangle$ app was found to grow linearly with absolute temperature $\mathrm{T}$, the ratio $\left\langle\mathrm{r}^{2}\right\rangle_{\text {app }} /(2 \mathrm{~T})$ entering the Debye-Waller factor being $0.000688 \AA^{2} / \mathrm{K}$. This value is comparable to the corresponding values of 0.00063 and $0.00103 \AA^{2} / \mathrm{K}$ reported for polyisobutylene ${ }^{35}$ and for polyisoprene, ${ }^{36}$ respectively.

Measurements were also carried out on the spectrometer IRIS (ISIS, Rutherford Appleton Laboratory, UK) using the same samples. The temperature range in these experiments was 340-460 K. QENS data were collected using the PG002 analyzer (resolution equal to $15 \mu \mathrm{eV}$ or $2.8 \times 10^{-10} \mathrm{~s}$ ) covering the energy range from -0.2 to $1.2 \mathrm{meV}$ and the $\mathrm{q}$ range between 0.55 and $1.81 \AA^{-1}$. Primary data were transformed

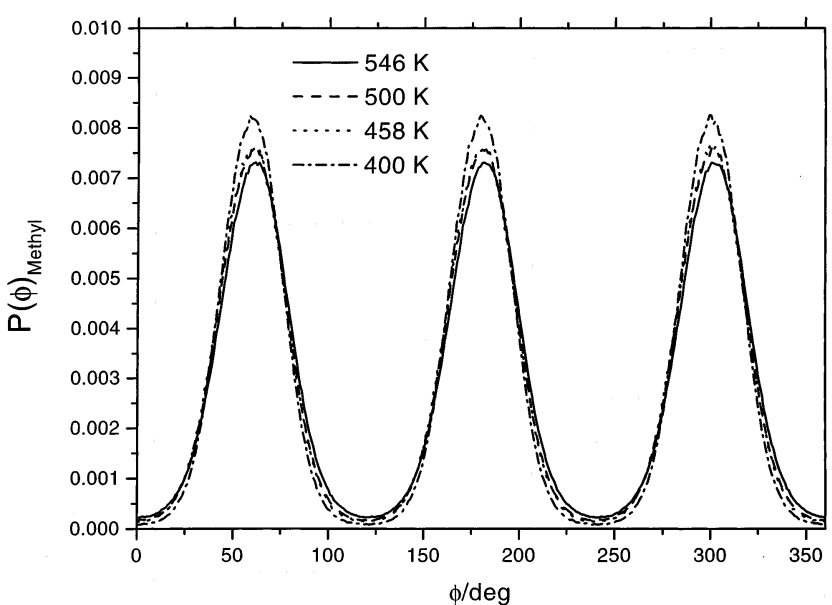

Figure 4. Probability distribution of dihedral angles $\phi$ describing rotation of methyls around their respective stems, as obtained from fully atomistic MD simulations using model I at three different temperatures. Angle $\phi$ is displaced by $\pi$ relative to the convention used in eq 1 .

into the time domain to obtain the incoherent intermediate scattering function I (q,t) using the FURY software ${ }^{34}$ available at ISIS, with due consideration of the instrumental resolution function.

Finally, some measurements were carried out on the NEAT instrument ${ }^{37}$ at BENSC in Berlin, Germany. There the wave length employed was $5.1 \AA$, leading to a resolution of $93 \mu \mathrm{eV}$ or $4.5 \times 10^{-11} \mathrm{~s}$ and covering the energy window between ca. -2.0 and $10 \mathrm{meV}$. The $\mathrm{q}$ range covered was between 0.5 and $2.25 \AA^{-1}$.

In all experiments, a slab cell was used, containing a 0.2 mm-thick sample of aPP. This corresponds to a transmission value equal to 0.9 , which ensures that multiple scattering effects are negligible.

\section{Methyl Group Conformation and Short-Time Dynamics As Revealed by Model I}

Distribution of Methyl Dihedral Angles. Figure 4 shows the probability distribution of the dihedral angles $\phi$ involving methyl hydrogens at four temperatures examined with model I. The expected three-fold symmetric distribution describing methyl rotation is clearly observed, in support of the form of the potential of mean force $\mathrm{V}(\phi)=-\mathrm{k}_{\mathrm{B}} \mathrm{T}$ In $\mathrm{P}(\phi)$ adopted in model II (eq 1).

Calculation of the associated potential barriers from Figure 4 as $E_{A}=-R T \ln \left(P_{\min } / P_{\max }\right)$, where $P_{\min }$ and $P_{\max }$ are the minimum and maximum values adopted by $\mathrm{P}(\phi)$, yields weakly temperature-dependent values with an average of $\mathrm{E}_{\mathrm{A}}=15.3 \pm 0.4 \mathrm{~kJ} / \mathrm{mol}$.

Distribution of Barrier Heights. To check whether a Gaussian form for the distribution of activation energies $\mathrm{E}_{\mathrm{A}}$ for methyl jumps is a reasonable assumption, we have fitted the time-distribution spectra extracted either from the intermediate incoherent scattering functions associated with methyl hydrogen displacements I $(q, t)$, or from the reorientation of the methyl $\mathrm{C}-\mathrm{H}$ bonds as expressed by the second-order correlation function

$$
P_{2}(t)=\frac{1}{2}\left\langle 3[\mathbf{u}(t) \cdot \mathbf{u}(0)]^{2}-1\right\rangle
$$

In eq 11 , $\mathbf{u}$ is the unit vector along the examined bonds.

As a first step, the dynamic spectra can be expressed as a superposition of exponential relaxation times according to the equation 


$$
C(t)=\int_{-\infty}^{+\infty} g(\ln \tau) e^{-t / \tau} d \ln \tau
$$

where $C(t)$ represents $I(q, t)$ or $P_{2}(t)$, and $g(\ln \tau)$ is the normalized (in the logarithmic scale) distribution of relaxation times (DRT). In this work, the CONTIN numerical procedure ${ }^{38}$ was used to derive $g(\ln \tau)$ in eq 12. The dynamic processes contributing to the decay of the examined function appear as distinct peaks in the $g(\ln \tau)$ representation. For symmetric distribution peaks, the location of the corresponding maxima is a good estimate of the characteristic time corresponding to each process. If a log-Gaussian expression of the form

$$
\mathrm{g}(\ln \tau)=\frac{\mathrm{RT}}{\sigma \sqrt{2 \pi}} \exp \left[-\frac{\mathrm{RT} \ln \left(\tau / \tau_{\mathrm{o}}\right)-\mathrm{E}_{\mathrm{o}}}{\sigma}\right]^{2}
$$

provides a satisfactory fit of the DRT, it can directly be related to a Gaussian distribution of potential barriers as expressed by eq 3, based on the reasonable assumption ${ }^{26,39}$ that the relaxation time $\tau$ shows an Arrhenius dependence on temperature:

$$
\tau(T)=\tau_{0} \exp \left(\frac{E_{A}}{k_{B} T}\right)
$$

In the above expression of $\mathrm{g}(\ln \tau), \mathrm{E}_{\mathrm{o}}$ corresponds to the average potential barrier, $\sigma$ is the width of the distribution, and $\tau_{0}$ is the inverse of an attempt jump frequency (cf. eq 7).

Incoherent Dynamic Structure Factor. The upper panel of Figure 5 shows the incoherent intermediate scattering function arising from the methyl hydrogens at $\mathrm{T}=400 \mathrm{~K}$ and at four different scattering vectors. The lines through the points indicate the fit provided by the DRT analysis. The lower panel displays the corresponding distributions of relaxation times. It must be noted that the subpicosecond regime, relevant to librational motions (see section 6 below), is not included in the present fit. I $\mathrm{n}$ the time window considered, at all the examined scattering vectors, the DRTs appear to be bimodal. The peak at longer times (on the order of hundreds of picoseconds) should be associated with contributions from sl ower backbone motions, while the faster peak can be ascribed to methyl motion. The latter assignment is supported by the q-independent peak location (i.e., characteristic time) of this process, as well as from its time scale, which is very close to the inverse of the independently calculated methyl jump rates (e.g., at the temperature shown, $\mathrm{T}=400 \mathrm{~K}$, the inverse methyl jump rate $2 \tau_{\text {jump }}$ for model II amounts to $20 \mathrm{ps}$ ). The line through the $q=1 \AA^{-1}$ DRT is an example of the fit by the log-Gaussian expression, eq 13. In this fit $\tau_{\text {o }}$ was fixed to a value of $28.8 \mathrm{meV}$ calculated experimentally from low-temperature neutron measurements (ref 26). The width $\sigma$ and average activation energy $\mathrm{E}_{\mathrm{o}}$ extracted from the fit are $\sigma=2.99 \pm 0.05$ $\mathrm{kJ} / \mathrm{mol}$ and $\mathrm{E}_{\mathrm{o}}=14.70 \pm 0.04 \mathrm{~kJ} / \mathrm{mol}$.

Reorientation of the Methyl $\mathbf{C}-\mathrm{H}$ Bonds in a Local Reference Frame. To minimize contribution from backbone motion in the calculated methyl spectra, so that comparison to low temperature neutron results (where the backbone is practically frozen) can be made, we have examined methyl reorientation as probed by $\mathrm{P}_{2}(\mathrm{t})$ in a local reference frame attached to the methyl group. The z-axis was chosen as the direction of the $\mathrm{C}-\mathrm{CH}_{3}$ bond, the x-axis was defined by the cross

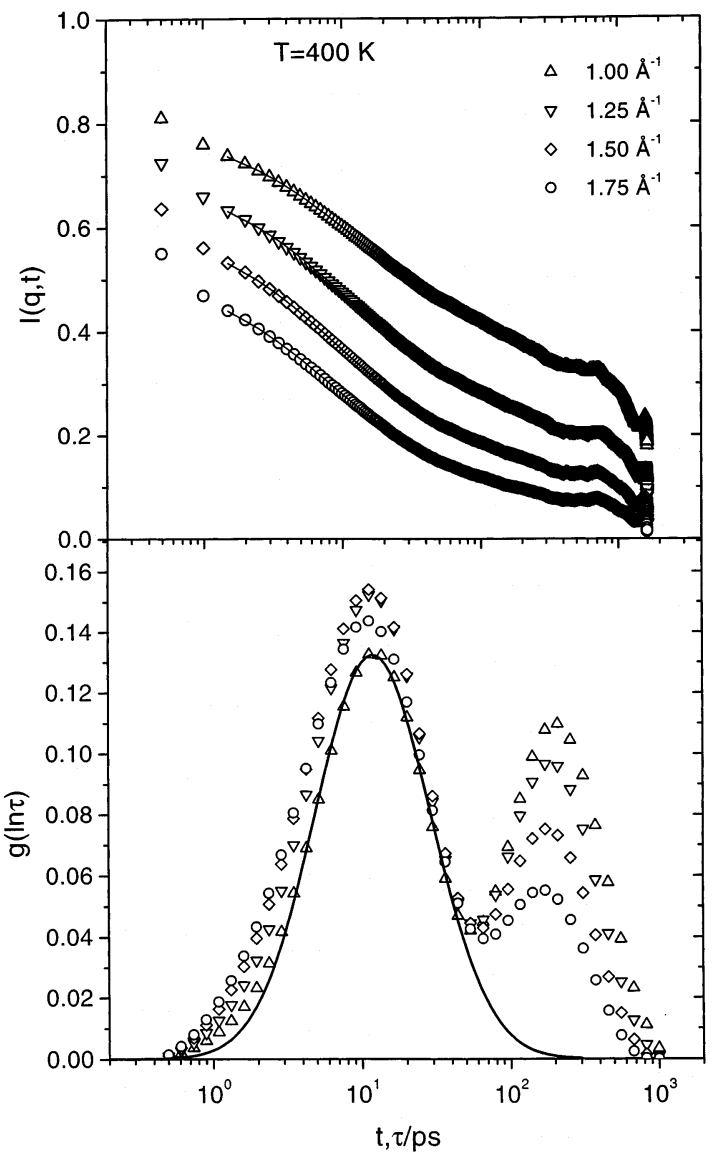

Figure 5. (top) Incoherent intermediate scattering function I (q,t), as calculated from model I at $400 \mathrm{~K}$. (bottom) Corre sponding distribution of relaxation times (DRT). Lines through the data points in the top figure are reconstructions of $I(q, t)$ from the DRT, using eq 12. Results are shown for four different $q$ values. The line in the bottom plot is a log-Gaussian fit to the DRT for $q=1 \AA^{-1}$ using eq 13.

product of the z-axis with the bisector of the $\mathrm{CH}_{2} \mathrm{CH}-$ $\mathrm{CH}_{2}$ bending angle, and the $y$-axis completed the local reference system. Figure 6 shows the $P_{2}(t)$ in the local reference frame at three temperatures, together with the corresponding fits (line through the points, upper panel) and the DRTs (lower panel). The thick lines through the DRTs trace the resulting log-Gaussian fits. The inset shows the extracted width of the distribution of activation energies, $\sigma$, as a function of temperature, while the corresponding average activation energies are listed as well. The width of the activation energy distributions appears to be insensitive to the temperature and slightly smaller than the value calculated from the fit to the I $\left(q=1 \AA^{-1}, t\right)$ at the same temperature (Figure 5). The average activation energies appear weakly temperature dependent but close to the $E_{0}$ calculated from the I $\left(q=1 \AA^{-1}, t\right)$ curve and to the value extracted from the neutron measurements (compare sections 3 and 4 ).

Degree of Correlation between Methyl J umps. To verify that there is no significant correlation in the rotation of different methyl groups, we have examined how the jump rate of methyls neighboring to a methyl group that undergoes a jump changes as a function of time elapsed from the triggering event. The rates were normalized to the uncorrelated average rate, so that only correlation-induced excess probability is monitored.

Figure 7 shows the degree of correlation observed at the three higher temperatures examined, for the two 


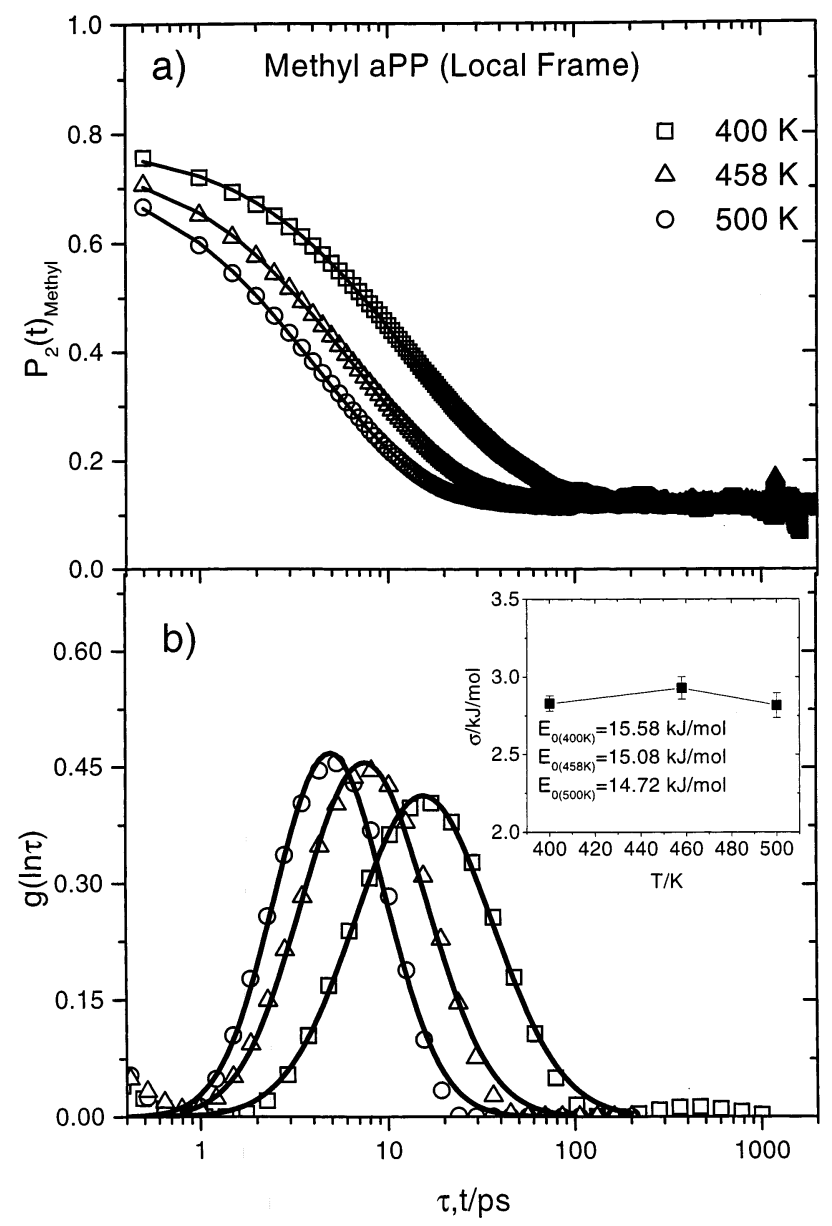

Figure 6. Analysis of the motion of methyl hydrogens but in the local reference frames of the methyls. (a, top) Orientational autocorrelation function $\mathrm{P}_{2}(\mathrm{t})$ of $\mathrm{C}-\mathrm{H}$ bonds. (b, bottom) DRT extracted therefrom using eq 12. Lines in the top plot are reconstructions of $\mathrm{P}_{2}(\mathrm{t})$ from the DRT. Results are shown at three different temperatures. The temperature dependence of mean values and standard deviations of activation energies extracted from the Gaussian fits are shown in the inset.

closest neighbors. A value of 1 for the ordinate corresponds to lack of correlation. Aside from a slightly enhanced probability close to the triggering jump, no significant excess correlation is observed, especially as the temperature rises (and statistics improves).

\section{Shape of the Relaxation Function, As Predicted from Simulations of Model II}

To compare MD predictions from model II with the neutron scattering data, the self-part of the Van Hove correlation function, $G_{s}(r, t)$ was calculated from the hydrogen trajectories obtained from the simulation. ${ }^{15}$ Then, the incoherent intermediate scattering function $\mathrm{I}(\mathrm{q}, \mathrm{t})$ was obtained by spatial Fourier transformation of $\mathrm{G}_{\mathrm{s}}(r, \mathrm{t})$.

In Figure 8, I $(q, t)$, as predicted from model II, is plotted as a function of time at a value of $q=0.767 \AA^{-1}$ in linear coordinates for five different temperatures. Separate curves are shown for the pendant hydrogens and for all the hydrogens (pendant and methyl). All curves exhibit a rapid decay at times less than 1 ps, which is attributable to fast bond angle vibrations and torsional librations (see bel ow). At $260 \mathrm{~K}$, the pendant hydrogens show very little further decorrelation beyond this fast initial drop. For the pendant hydrogens at 260 $\mathrm{K}$, and even at $300 \mathrm{~K}, \mathrm{I}(\mathrm{q}, \mathrm{t})$ up to times of $300 \mathrm{ps}$ looks

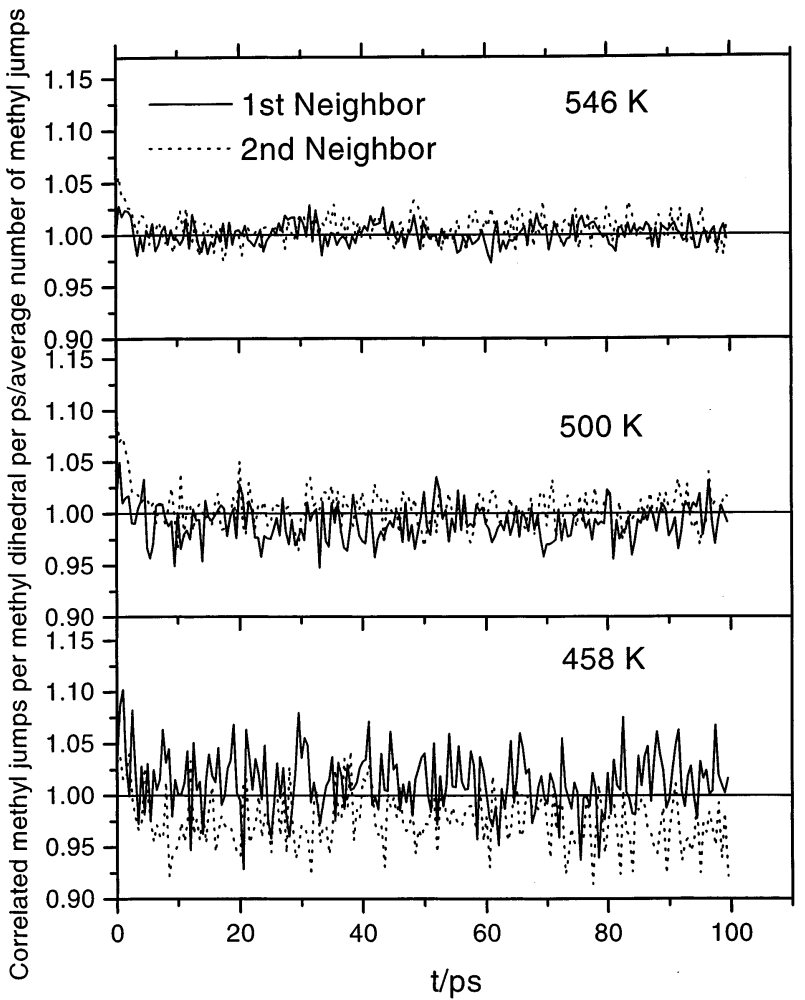

Figure 7. Normalized number of torsional jumps executed by 1st and 2nd neighbors of a reference methyl as a function of time elapsed from a jump of the reference methyl. A value of 1 corresponds to complete lack of correlation. Results are shown for three different temperatures.

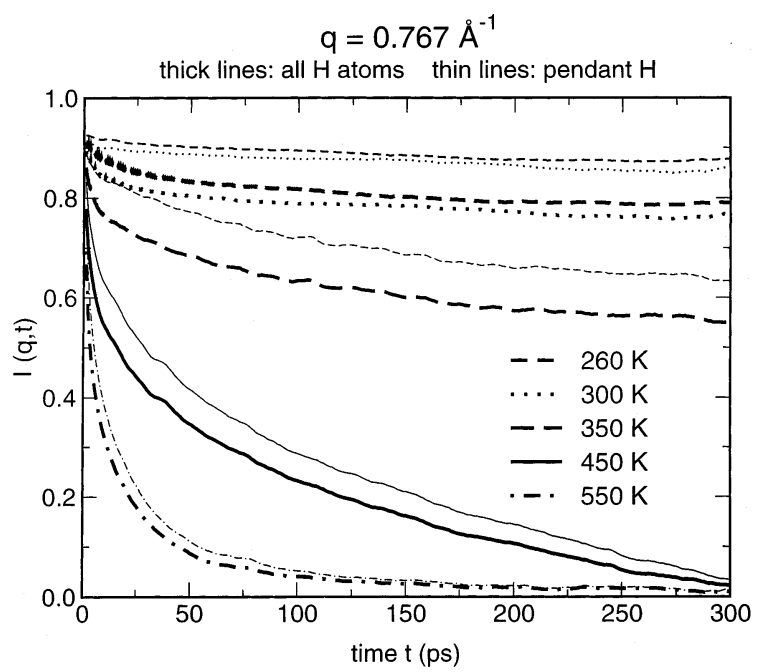

Figure 8. I ncoherent intermediate scattering functions I (q,t) of pendant hydrogens (thin lines) and of all hydrogens (thick lines) at fixed $q=0.767 \AA^{-1}$, as obtained from the simulations (model II) at five different temperatures $(260,300,350,450$, and $550 \mathrm{~K}$ ).

very much like a horizontal line in the coordinates of Figure 8; the dependence on $t$ is very weak. Total hydrogen curves at 260 and $300 \mathrm{~K}$ display a significant decay between 1 and ca. 100 ps and then stay more or less parallel to the corresponding pendant hydrogen curves. This decay is related to torsional jumps of the methyl groups; for comparison, the mean residence time $1 /\left(2 k^{T S T}\right)$ within a methyl energy well is ca. 52 ps at 260 $\mathrm{K}$ (see section 3 above) and ca. 22 ps at $300 \mathrm{~K}$. At the higher temperatures of 350, 450, and $550 \mathrm{~K}$, backbone torsional librations become larger in amplitude and 


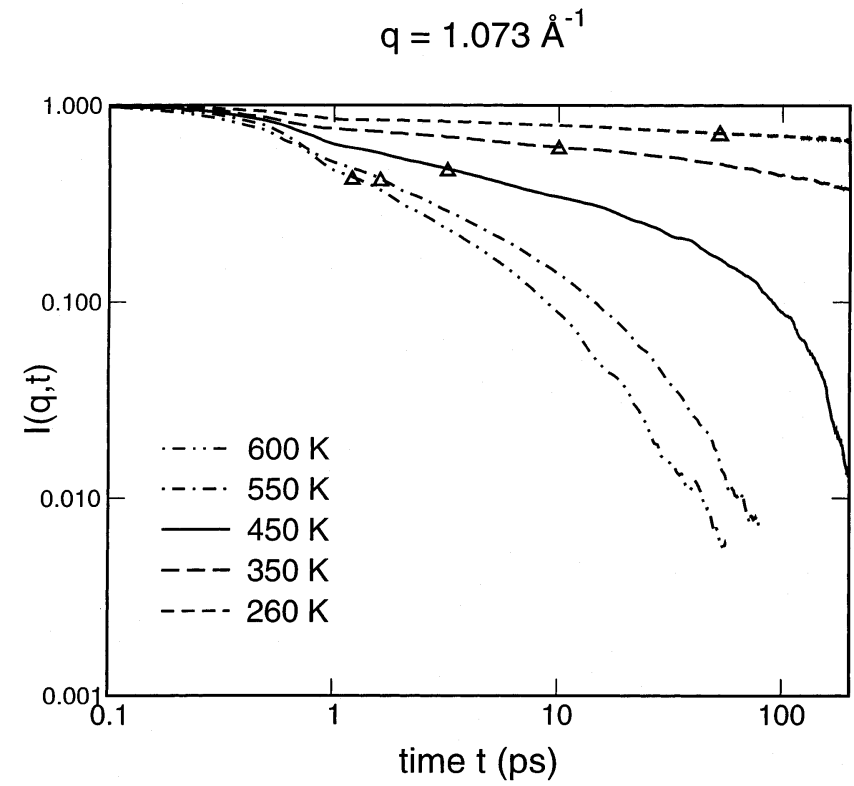

Figure 9. Incoherent intermediate scattering function I (q,t) of all hydrogens at fixed $q=1.073 \AA^{-1}$, as obtained from the simulations (model II) at five different temperatures $(260,350$, 450, 550, and $600 \mathrm{~K}$ ), plotted against time in logarithmic coordinates. The typical residence times $\tau_{\text {jump }}$ between torsional transitions of methyl groups are indicated by the triangles at each temperature.

conformational transitions of the backbone set in and become widespread, leading to rapid decay of I $(q, t)$. The difference in I $(q, t)$ between pendant and total hydrogens diminishes as temperature is raised. At $550 \mathrm{~K}$, almost complete decorrelation is seen for all types of hydrogens at the highest correlation time of 300 ps.

In Figure 9, I (q,t) from all hydrogens is again plotted as a function of $\mathrm{t}$ for various temperatures at a value of $q=1.073 \AA$, but this time in logarithmic coordinates, to emphasize short-time behavior. In these coordinates a continuous decay of I (q,t) is seen at all temperatures, including $260 \mathrm{~K}$. All curves, and especially the three higher temperature ones, show some structure. One sees a short-time hump in the curves, which is followed by a more or less linear region leading into the long-time " $\alpha$ " relaxation process. The bend between the short-time hump and the quasilinear region occurs at a time around 1 ps for all temperatures, suggesting that vibrational and librational processes, and not activated transitions, are responsible for the hump. For comparison, the typical residence times of methyl groups in their wells between torsional transitions, $\tau_{\text {jump }}$, have been marked on the curves of Figure 9 as open triangles. It is seen that these times fall in the quasilinear region beyond the short-time relaxation hump, confirming that the short-time process is due to bond angle vibrations and torsional librations of the skeletal bonds and not to torsional transitions of any kind.

Figure 10 presents closeups of the decay of $I(q, t)$ with $t$ at three values of the wavevector $q$, in logarithmic coordinates. The observations made above in conjunction with Figure 4 about the shape of the relaxation function are confirmed. The time scale of the short-time hump changes very little with temperature and the crossover between the hump and the longer time decay of $I(q, t)$ always occurs at 1 ps, independently of $q$. The methyl torsional transition time $\tau_{\text {jump }}$, marked by the vertical bars on the abscissa, clearly falls outside the a
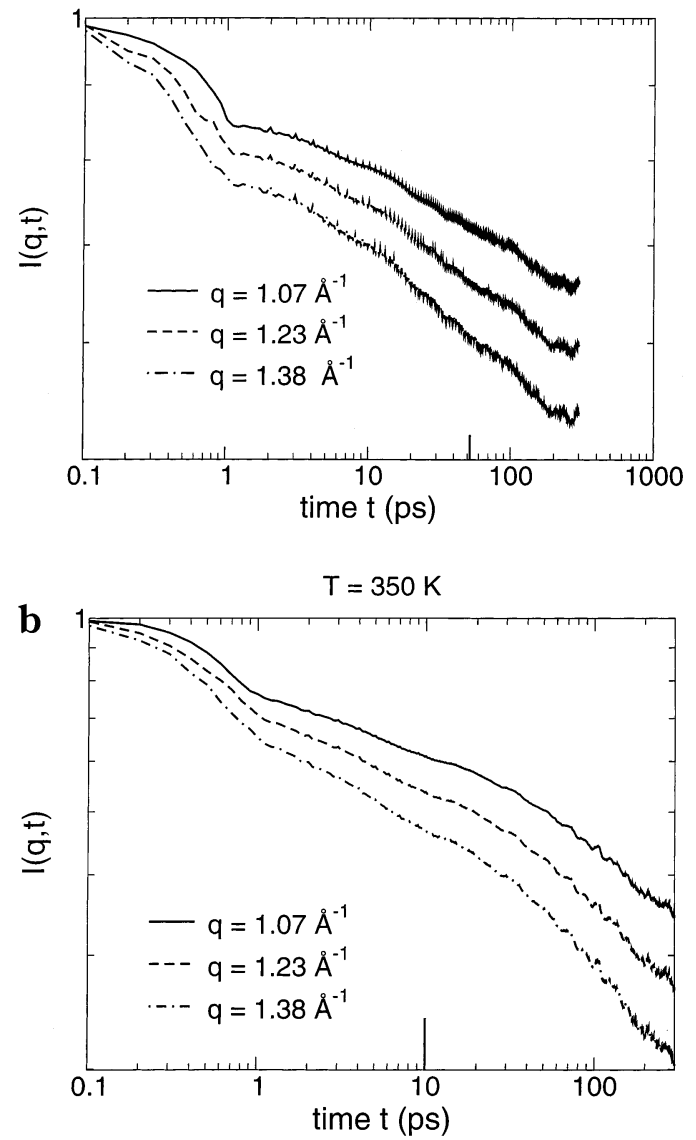

Figure 10. Incoherent intermediate scattering function I (q,t) plotted as in Figure 9, but at three values of $q(1.07,1.23,1.38$ $\AA^{-1}$ ). (a) $\mathrm{T}=260 \mathrm{~K}$, (b) $\mathrm{T}=350 \mathrm{~K}$. The thick bar on the abscissa marks $\tau_{\text {jump. }}$

hump region. The "fine structure" seen in the $260 \mathrm{~K}$ plot is an artifact of the simple way in which methyl torsional librations are represented in model II (perfectly harmonic torsional oscillations; see section 3).

In Figure 11 the $\log [I(q, t)]$ versus log t curve at $T=$ $450 \mathrm{~K}$ has been separated into contributions from methyl hydrogens and from hydrogens directly attached to the backbone. Results are shown for $\mathrm{q}=2.15 \AA^{-1}$ (Figure $11 \mathrm{a}$ ) and for $\mathrm{q}=3.4 \AA^{-1}$ (Figure $11 \mathrm{~b}$ ). In the range $0.1 \mathrm{ps}<\mathrm{t}<1 \mathrm{ps}$, we see that the decorrelation is appreciably greater for methyl hydrogens than for pendant hydrogens. This is because, in this time range, pendant hydrogens decorrelate solely through bond angle bending vibrations and torsional librations of the skeletal bonds, while methyl hydrogens participate, additionally, in torsional librations of the methyl groups, of period ca. $0.14 \mathrm{ps}$. In the range $1 \mathrm{ps}<\mathrm{t}<10 \mathrm{ps}$ we see that the gap between methyl and pendant hydrogen curves in Figure 11 widens. In this range, torsional transitions of the methyls set in (again, values of $\tau_{\text {jump }}$ have been marked on the plots). These transitions are clearly responsible for the faster, quasilinear decay of methyl curves observed in this region. It is noteworthy that, although the total hydrogen relaxation curve at $\mathrm{q}$ $=2.15 \AA$ appears featureless from 0.1 to $10 \mathrm{ps}$, its methyl part does exhibit structure due to the methyl jumps. At times beyond $30 \mathrm{ps}$, torsional transitions of skel etal bonds become widespread and one observes the precipitous drop characteristic of " $\alpha$ " relaxation in the coordinates of Figure 11a. 

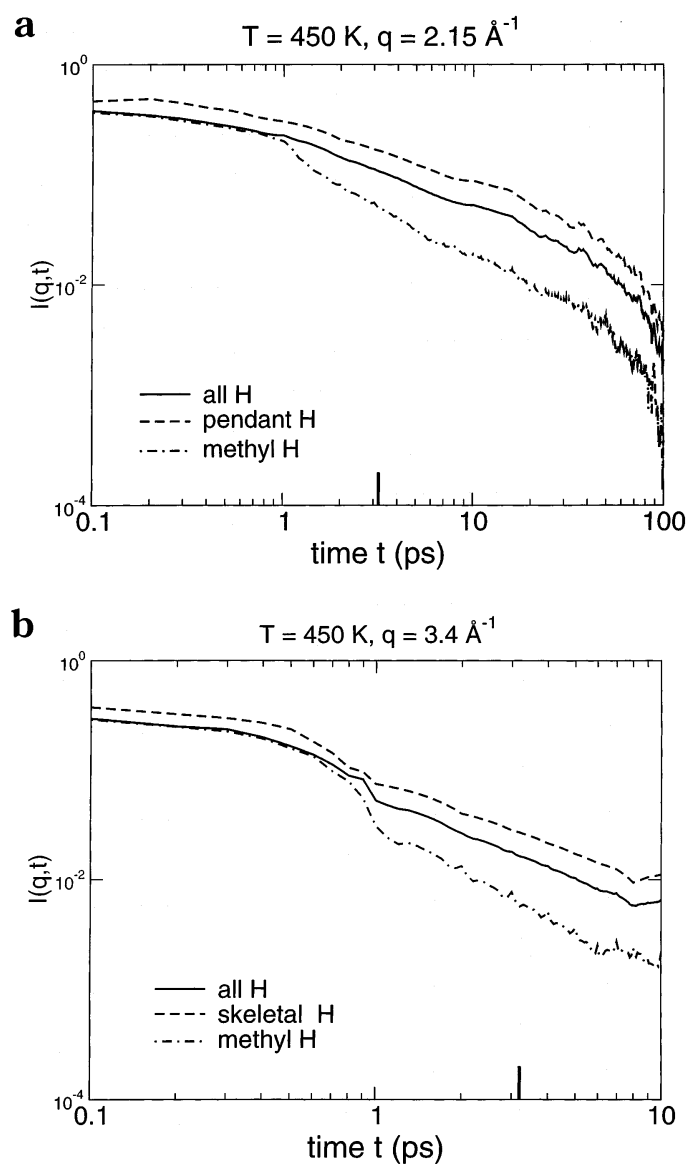

F igure 11. I ncoherent intermediate scattering function I (q,t) at $\mathrm{T}=450 \mathrm{~K}$ plotted as a function of time in logarithmic coordinates. Both the total function and the individual contributions from pendant hydrogens and methyl hydrogens are shown: (a) $q=2.15 \AA^{-1}$, (b) $q=3.4 \AA^{-1}$. The thick bar on the abscissa indicates $\tau_{\text {jump }}$.

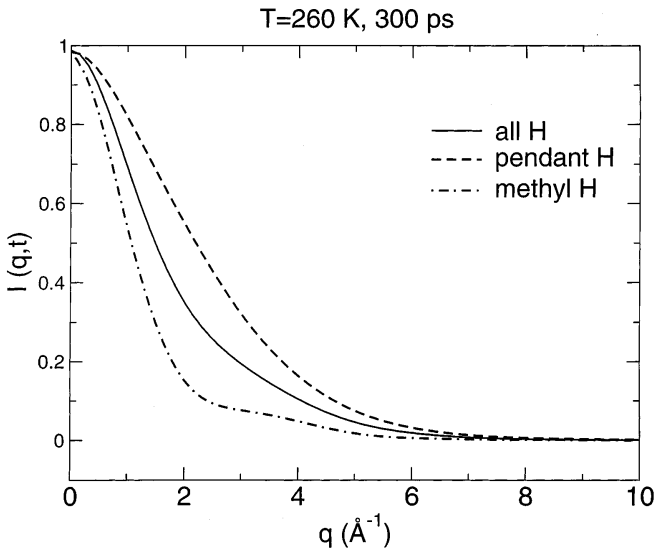

Figure 12. I ncoherent intermediate scattering function I (q,t) from the simulation (model II) as a function of $q$ for a time of $t=300$ ps at $260 \mathrm{~K}$, separated into contributions from hydrogens pendant to the backbone and from hydrogens belonging to methyl groups.

Interestingly, the q-dependence of I, as cal culated on the basis of model II, is found to depart somewhat from the Gaussian behavior $\left[I \approx \exp \left(-\mathrm{q}^{2}\left\langle\mathrm{r}^{2}(\mathrm{t})\right\rangle\right)\right]$ often postulated for it. To examine this departure more closely, we show I (q,t) as a function of $\mathrm{q}$ at $\mathrm{T}=260 \mathrm{~K}$ for $\mathrm{a}$ correlation time $t=300 \mathrm{ps}$ in Figure 12. The components of $I(q, t)$ coming from hydrogens pendant to the backbone and from hydrogens belonging to methyl groups are shown separately in this figure. As discussed

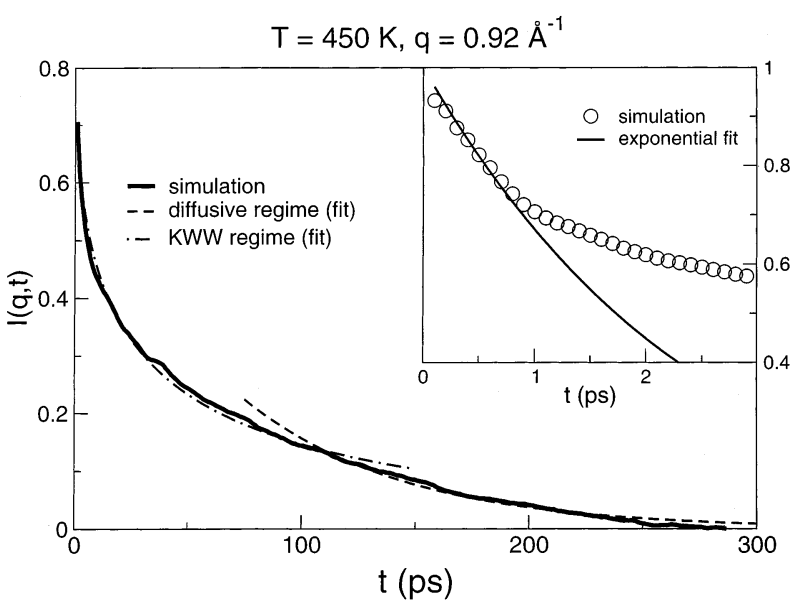

Figure 13. Time-dependence of the intermediate scattering function I $(\mathrm{q}, \mathrm{t})$ as obtained from the simulation at $\mathrm{T}=450 \mathrm{~K}$ and $q=0.92 \AA^{-1}$, and its analytical approximation with two exponential and a stretched exponential function. Inset shows the exponential fit at short times.

above, methyl hydrogens decorrelate faster than pendant hydrogens. WhileI (q,t) for the pendant hydrogens has the usual Gaussian-like shape, I (q,t) for the methyl groups exhibits a clear shoulder peak at approximately $3.5 \AA^{-1}$. This peak is an immediate consequence of wellto-well jumps of the methyls. According to our molecular model, the distance traversed by a methyl hydrogen during such a jump is $1.10 \sin \left(70^{\circ}\right) 2 \sin \left(60^{\circ}\right) \AA=1.79$ $\AA$, corresponding to a q value of $2 \pi /(1.79 \AA)=3.5 \AA^{-1}$.

In Fourier transforming I (q,t) with respect to time and comparing against experimental QENS spectra, and al so in computing correlation times from the simulation, it is useful to describe the time dependence of I $(q, t)$ via analytical expressions. As exemplified by Figure 13, which corresponds to $T=450 \mathrm{~K}$ and $\mathrm{q}=0.92 \AA^{-1}$, computed I $(\mathrm{q}, \mathrm{t})$ curves can be fit reasonably well by three analytical expressions. The first is a fast-decaying exponential, which describes the initial decorrelation at times up to $1 \mathrm{ps}$, for which the observed dynamics is governed by bond angle bending vibrations and torsional librations. The time constant of this exponential was found equal to 0.85 and 0.78 ps at $q=1.38 \AA^{-1}$ and $q=$ $2.3 \AA^{-1}$, respectively.

This nearly exponential decay of I (q,t) at subpicosecond time scales has been reported for a variety of glassforming systems, including silica melts, ${ }^{40}$ at high temperatures relative to $T_{g}$, on the basis of both experimental and simulation investigations. At a time on the order of $0.01 \mathrm{ps}$, the translational dynamics of particles crosses over from a ballistic regi me (displacement proportional to time) to a relaxation behavior, which is basically exponential up to time scales of approximately 1 ps. The microscopic picture ${ }^{40}$ behind this short-time exponential decay is one of particles rattling within a "cage" formed by their surrounding particles, the walls of the cage themselves being displaced, owing to the motion of the surrounding particles. In low-molecular weight liquids at high temperatures, this exponential decay corre sponds to particle self-diffusion and persists until I (q,t) has fallen essentially to zero. ${ }^{40}$ Closer to the glass transition, and especially in the presence of long-range connectivity, the initial exponential decay gives way to a slower decay, as particles sense the hierarchical constraints inherent in the system dynamics. As shown in the inset of Figure 13, a single-exponential fits the dynamics in the region $t<1$ ps satisfactorily, but not 


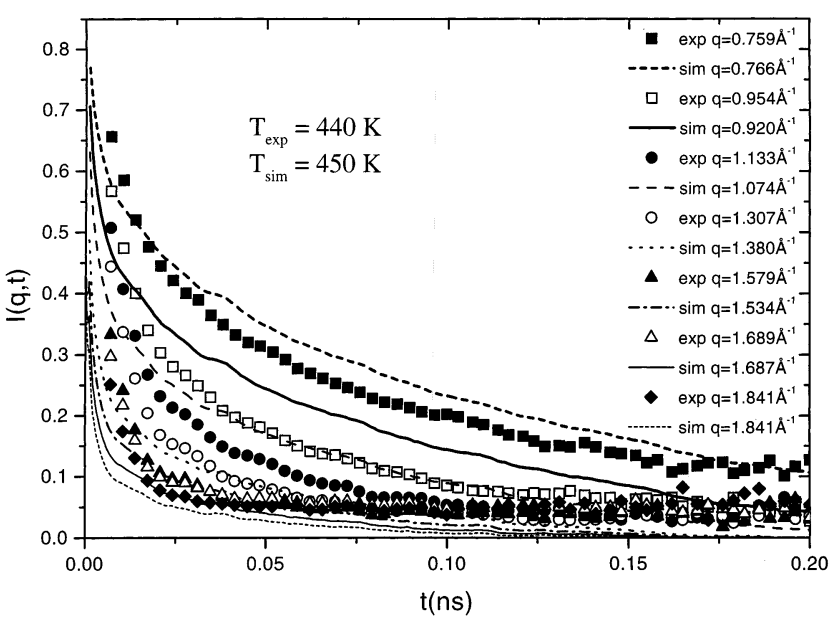

Figure 14. Simulated (lines) and experimental (points) I (q,t) at various $q$ values. The temperature is $440 \mathrm{~K}$ in the experiment and $450 \mathrm{~K}$ in the simulation.

perfectly; the very short-time decay of the computed curve is faster, owing to the fast torsional oscillations of the methyl groups, of period ca. 0.14 ps, as discussed above.

Beyond 1 ps, the decay of I (q,t) is clearly broader than can be captured by a single exponential. As shown in Figure 13, the region from 1 to 100 ps is well-described by a stretched exponential (Kohlrausch-WilliamsWatts, KWW) function of the form

$$
\mathrm{I}_{\mathrm{KWw}}(\mathrm{q}, \mathrm{t})=\operatorname{const}(\mathrm{q}) \exp \left[-\left(\mathrm{t} / \tau_{\mathrm{KWw}}(\mathrm{q})\right)^{\beta}\right]
$$

At very long times, longer range modes of motion (Rouse modes, self-diffusion of entire chains) come into play. This long-time decay of I (q,t), which is accessible by the simulation only at high temperatures, is again described better by a single exponential, rather than by a stretched exponential (see Figure 13). Thus, I (q,t) can generally be described piecewise by three continuous functions.

The piecewise fit of the computed I $(q, t)$ by three functions (an exponential, a KWW function, and then another exponential), described above, has been used in calculating correlation times $\tau_{\mathrm{c}}$ [by integration of I $(\mathrm{q}, \mathrm{t})]$, stretching exponents $\beta$, and incoherent dynamic structure factors $\mathrm{S}(\mathrm{q}, \omega)$ [by Fourier transformation of $\mathrm{I}(\mathrm{q}, \mathrm{t})]$ in the following.

To enable comparisons with QENS measurements and simulations conducted by other investigators, a second, simpler fitting procedure has also been adopted. In this procedure, the entire long-time decay of $I(q, t)$ beyond 1 ps is fitted by a single KWW function (eq 15), i.e., no exponential long-time tail is distinguished for I $(q, t)$. In the following sections, values of the KWW time obtained through this simpler fitting procedure will be referred to as $\tau_{\mathrm{K} w \mathrm{w}}$, and observations made on these values will be compared to those of other investigators.

\section{Comparison between Simulation (Model II) and QENS Experiments}

Figure 14 shows a direct comparison between incoherent intermediate scattering functions, I $(q, t)$, as computed from model II and as extracted from the ISIS measurements. The temperatures are $440 \mathrm{~K}$ in the experiment and $450 \mathrm{~K}$ in the simulation. Results are presented for a range of $q$ values, the $q$ values used in the calculation being very close to those of the experi-

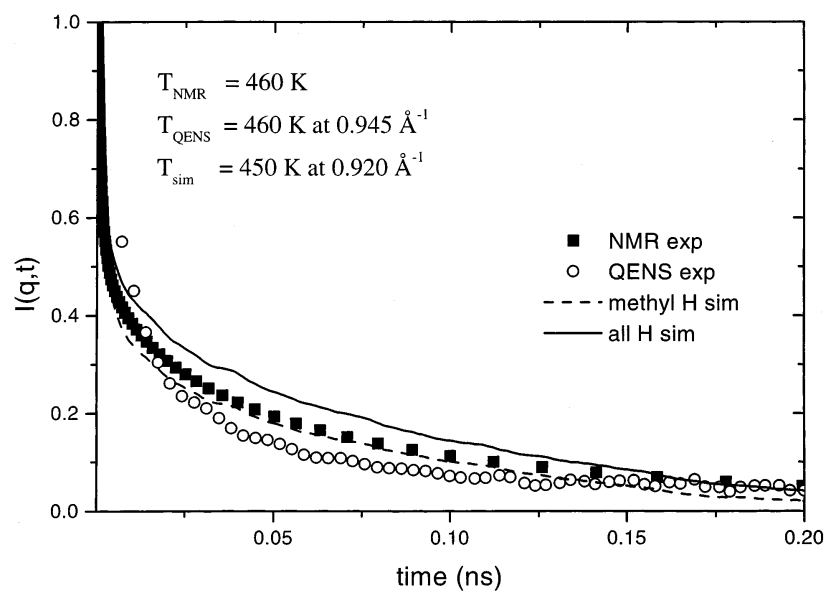

Figure 15. Simulated $I(q, t)$ at $T=450 \mathrm{~K}$ and $q=0.92 \AA^{-1}$ (lines) being compared to the experimental I $(\mathrm{q}, \mathrm{t})$ at $\mathrm{T}=460$ $\mathrm{K}$ and $\mathrm{q}=0.945 \AA^{-1}$ and to an orientational relaxation function $\mathrm{P}_{2}(\mathrm{t})$ from NMR at $\mathrm{T}=460 \mathrm{~K} .{ }^{21}$ Two curves are shown for the simulated I (q,t), one for all the hydrogens and one for methyl hydrogens only.

ment in most cases. Agreement between simulation and experiment is generally good. The simulation tends to overpredict the decay rate of $\mathrm{I}(\mathrm{q}, \mathrm{t})$ at short times and to underpredict it at long times. In all cases, the predicted correlation time (integral between I (q,t) and its long-time asymptote) is within a factor of 2 from its experimental counterpart. Comparable agreement was found for correlation times measured by NMR and simulated using the same model.3,41,21 At long times, the experimental I $(q, t)$ curves approach a nonzero plateau, suggesting an "elastic" contribution to the scattering that does not relax over the time scale of Figure 14. This is especially evident in the low-q experimental results. It is absent from the simulation results, which decay to zero at long times.

It is often stated that QE NS data at a q value around $1 \AA^{-1}$ should give a relaxation behavior comparable to that measured by NMR relaxation experiments. ${ }^{42}$ To test this idea, we compare in Figure 15 the QENS relaxation function I $(\mathrm{q}, \mathrm{t})$, as computed from our simulations at $\mathrm{T}=450 \mathrm{~K}$ and $\mathrm{q}=0.92 \AA^{-1}$ and as measured experimentally at ISIS at T $=460 \mathrm{~K}$ and $\mathrm{q}=0.945 \AA^{-1}$, against the NMR relaxation function $P_{2}(t)$ reported by Moe et al. ${ }^{21}$ for molten aPP. $P_{2}(t)$, defined as in eq 11 , is an orientational correlation function for pendant $\mathrm{C}-\mathrm{H}$ bonds belonging to methylene groups of the chains. Direct comparisons between $\mathrm{P}_{2}(\mathrm{t})$ as measured experimentally and as computed from MD simulations of model II have been reported ${ }^{3,21}$ and will not be repeated here. The $\mathrm{P}_{2}(\mathrm{t})$ points given in Figure 15 have been calculated from the analytical expression and parameters reported by Moe et al. in ref 21 , which provide an excellent fit to their NMR relaxation data. Calculated QENS curves are given both for all hydrogens and for methyl hydrogens only. Clearly, all sets of data shown in Figure 15 are reasonably close together. I nterestingly, the computed QENS relaxation curve lies closer to the NMR than to the experimental QENS data.

Correlation times for segmental motion, $\tau_{\mathrm{c}}(\mathrm{q})$, have been computed from the simulated I $(q, t)$ curves as

$$
\tau_{c}(q)=\int_{0}^{\infty} I(q, t) d t
$$

The logarithm of $\tau_{\mathrm{c}}\left(1 \AA^{-1}\right)$ is plotted against inverse temperature in Figure 16. Two sets of simulation results 


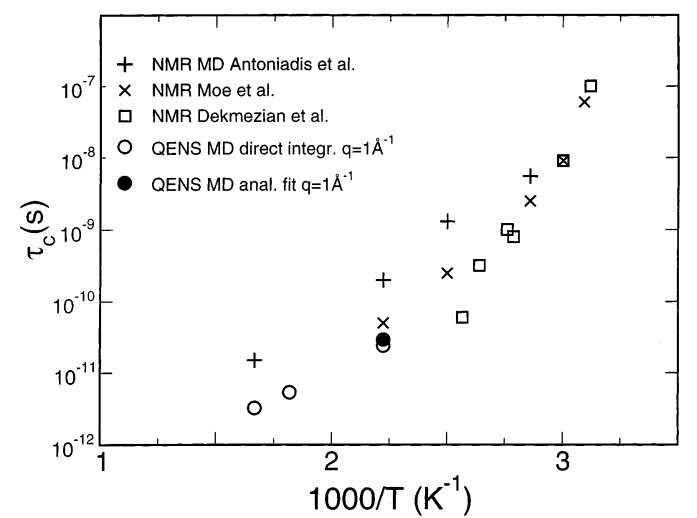

Figure 16. Temperature dependence of the correlation time extracted from the time decay of the simulated I $(q, t)$ at $q=1$ $\AA^{-1}$ being compared to the corresponding dependence of experimental (refs 21, 41) and simulated (ref 3) NMR correlation times. Open circles: correlation times calculated by direct numerical integration of the computed I $(q, t)$. Filled circle: correlation time calculated from fitted analytical approximation to the computed I $(q, t)$.

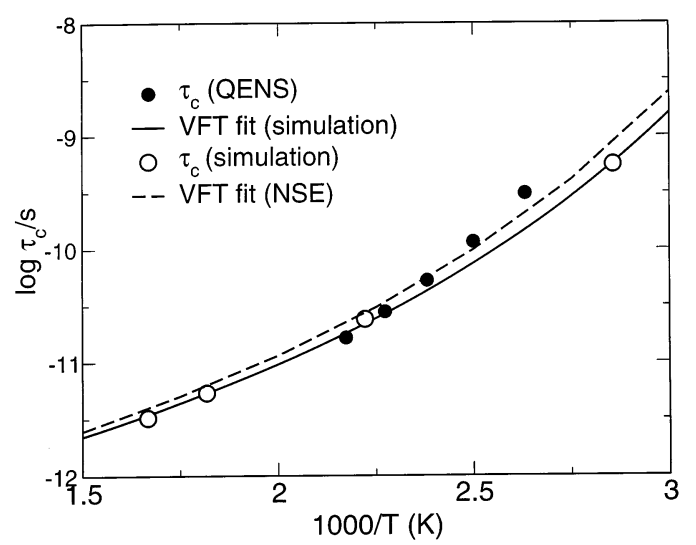

Figure 17. Temperature dependence of the correlation time $\tau_{\mathrm{c}}$ extracted from the time decay of the simulated $\mathrm{I}(\mathrm{q}, \mathrm{t})$ at $\mathrm{q}=$ $1 \AA^{-1}$ (open circles), al ong with its fit via a VFT function (solid line). Correlation times extracted from analysis of the QENS measurements at $q=1 \AA^{-1}$ are also shown for comparison (filled circles), as is a VFT fit to correlation time values obtained from coherent NSE measurements ${ }^{43}$ at $q=1 \AA^{-1}$ (broken line).

are given: Open symbols have been obtained by direct numerical integration of the I $(q, t)$ function computed from the simulation. The filled symbol at $450 \mathrm{~K}$ has been obtained by integrating the analytical representation of $\mathrm{I}(\mathrm{q}, \mathrm{t})$, consisting of exponential and $\mathrm{KWW}$ functions, as described above. The deviation between open and filled symbols at $450 \mathrm{~K}$ provides a measure of the accuracy with which $\tau_{\mathrm{c}}\left(1 \AA^{-1}\right)$ can be computed from simulation. Also in Figure 16 we have included results for the NMR correlation time

$$
\tau_{\mathrm{c}}=\int_{0}^{\infty} \mathrm{P}_{2}(\mathrm{t}) \mathrm{dt}
$$

as computed from MD simulations using model $\mathrm{II}^{3}$ and as measured experimentally. ${ }^{41,21}$ The different sets of data are in reasonable agreement, exhibiting a distinctly non-Arrhenius temperature dependence.

In Figure 17 the logarithms of correlation times $\tau_{\mathrm{c}}(\mathrm{q})$ calculated through eq 16 from the simulation at a value of $q=1 \AA^{-1}$ are plotted as a function of inverse temperature (open circles). The solid line through the simulation points is a best fit with a Vogel-Fulcher-

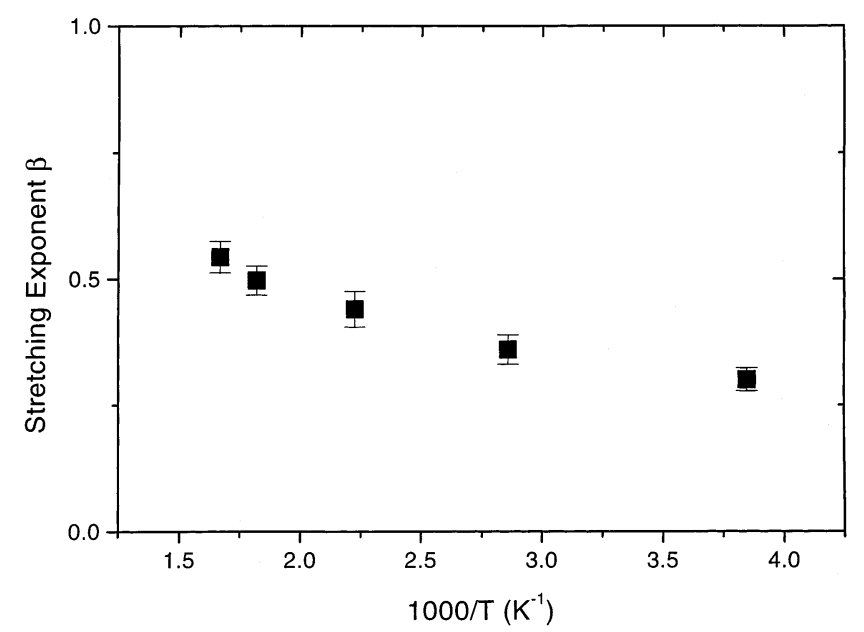

Figure 18. Stretching exponent $\beta$ extracted from KWW fit of $\mathrm{I}(\mathrm{q}, \mathrm{t})$ as a function of temperature.

Tammann (VFT) function, of the form

$$
\log \tau_{\mathrm{c}}=\log \tau_{\infty}+\frac{\mathrm{B}}{\mathrm{T}-\mathrm{T}_{\infty}}
$$

which is seen to capture the temperature dependence of $\tau_{\mathrm{c}}$ very well. On the same plot are shown values of the correlation times extracted from the QENS measurements at ISIS at $q=1 \AA^{-1}$ (solid circles). Also shown (broken line) is a VFT fit of the temperature dependence of $\tau_{\mathrm{c}}$ values determined by Arrighi et al.43 through (coherent) NSE measurements on deuterated aPP of mean molar mass $2570 \mathrm{~g} / \mathrm{mol}$ and narrow chain length distribution at $q=1.1 \AA^{-1}$ in the temperature range $300-360 \mathrm{~K}$. Clearly, all sets of $\tau_{\mathrm{c}}$ data are in very reasonable agreement. The temperature dependence of the simulation points parallels that of the NSE data very well, while the temperature dependence of the QENS data seems somewhat stronger.

Figure 18 presents the stretching exponent $\beta$ providing the best KWW fit to the middle part of the incoherent intermediate scattering function I $(q, t)$ from simulation, determined as discussed in conjunction with Figure 13. $\beta$ is seen to decrease with decreasing temperature from a value of 0.54 at $600 \mathrm{~K}$ to 0.3 at $260 \mathrm{~K}$. In the same temperature range, the QENS data from ISIS (Figure 14) can be fitted using a KWW function with $\beta=0.34-0.44$, which is a decreasing function of temperature, and an elastic component, convoluted in $\omega$-space with the instrumental resolution. These values of $\beta$ are reasonably consistent with values in the range $0.36-0.39$ determined from past MD simulations of the orientational decorrelation of pendant bonds in aPP. ${ }^{3}$ On the other hand, fitting the experimental data with a sum of a fast exponential decay and a KWW function leads to a value of $\beta \approx 0.50$, and a similar fit to NSE measurements ${ }^{43}$ in the range $300-360 \mathrm{~K}$ leads to a more or less temperature-independent value of $\beta=0.52$. Clearly, the value of the stretching exponent extracted from the data depends on how exactly the fit is performed.

The q-dependence of correlation times $\tau_{c}(q)$ determined from the simulated I $(q, t)$ through eq 16 is shown in Figure 19 in logarithmic coordinates. All points shown in the figure were determined by direct numerical integration of I (q,t); no analytical extrapolation was used. F or the numerical integration to be reliable, I (q,t) 


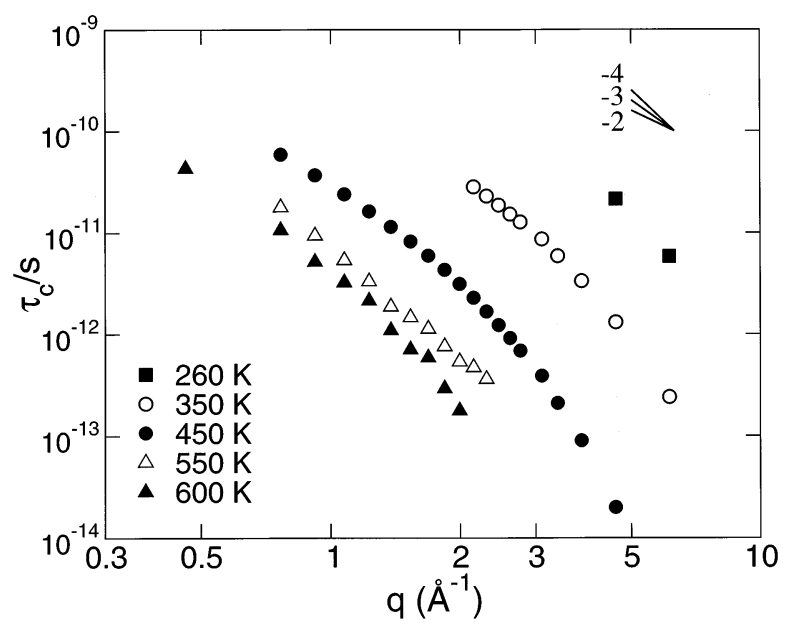

Figure 19. q-dependence of correlation times extracted from the simulated I (q,t) at various temperatures, in logarithmic coordinates.

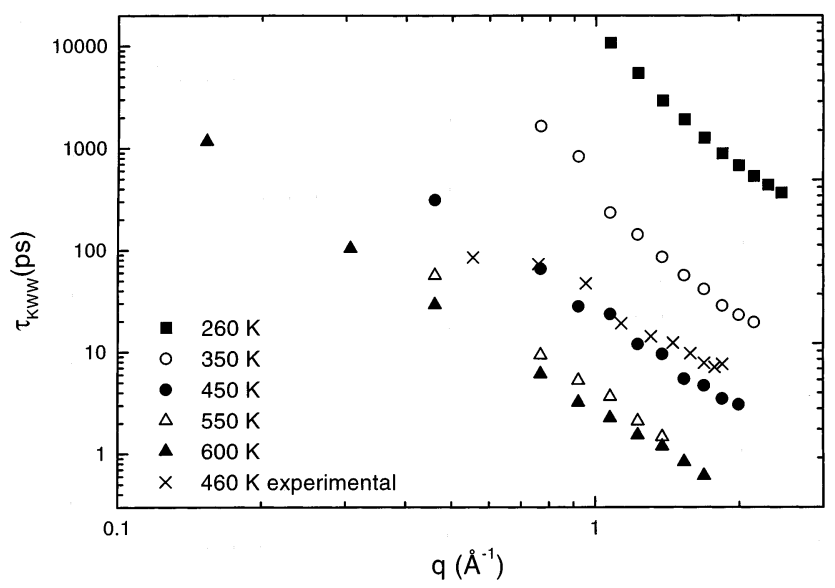

Figure 20. Characteristic time $\tau_{\mathrm{K} w w}$, obtained by fitting the long-time decay of I $(q, \mathrm{t})$ of all hydrogens in the system by eq 15, plotted against $q$ for five different temperatures. Experimental values at $460 \mathrm{~K}$ are also shown.

should have fallen to zero within the observation time of the simulation; this explains why points corresponding to low temperatures are restricted to high q values in Figure 19. In the same figure, straight lines with slopes of $-4,-3$, and -2 are indicated, for comparison. At all temperatures, the exponent $m$ in the scaling relation $\tau_{\mathrm{c}} \propto \mathrm{q}^{-\mathrm{m}}$ decreases with decreasing $\mathrm{q}$ (increasing length scale of observation). At large q (small-length scale motion) $m$ assumes a value of 4 or higher. At smaller q (larger length scale of observation) the $\tau_{\mathrm{c}}(\mathrm{q})$ curves bend toward a weaker $q$ dependence, $m$ being closer to 3. A likely explanation of this value of the exponent is that self-diffusion of entire chains in the (small molecular weight) model system starts contributing to the dynamics, driving the dependence toward the value $m=2$ that is characteristic of diffusional processes.

In Figure 20, the q-dependence of $\tau_{\mathrm{Kww}}$ values, extracted through a single KWW fit to the long-time decay of $I(q, t)$ beyond $1 \mathrm{ps}$, is displayed for the motion of all hydrogens in the system at five different temperatures. Understandably, the curves have a considerably different appearance from the corresponding curves of Figure 19, especially at high q values, where the correlation time $\tau_{c}$ is influenced significantly by the initial fast exponential decay of $\mathrm{I}(\mathrm{q}, \mathrm{t})$. Experimental values of $\tau_{\mathrm{KWw}}$ from the ISIS measurements at $460 \mathrm{~K}$

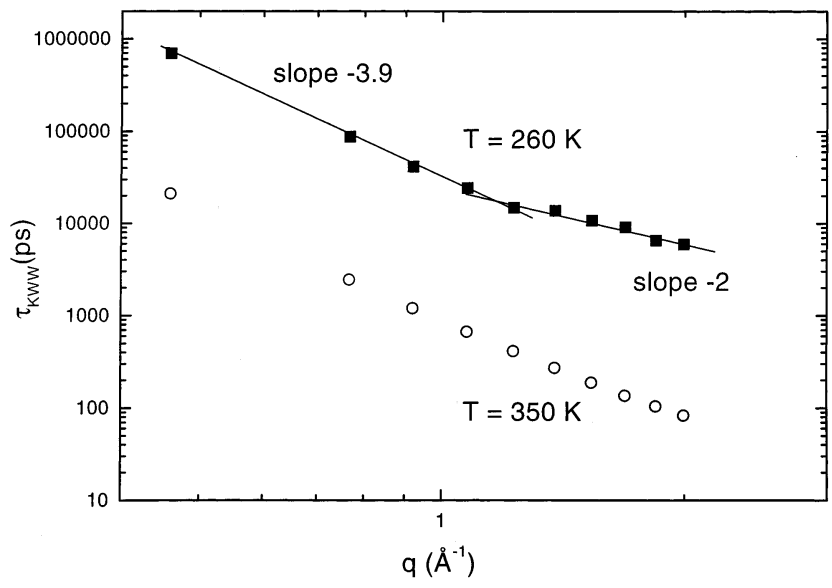

Figure 21. Characteristic time $\tau_{\mathrm{Kww}}$, obtained by fitting the long-time decay of I $(q, \mathrm{t})$ for the pendant hydrogens only by eq 15 , plotted against q for two different temperatures.

are al so shown. They are seen to agree very well with the $\mathrm{T}=450 \mathrm{~K}$ simulation results.

Figure 21 displays similar $\tau_{\mathrm{Kww}}(\mathrm{q})$ plots, calculated from the motion of pendant hydrogens only in the course of the MD simulations. In their MD simulation study of polyisoprene at $363 \mathrm{~K}$, Colmenero et al. ${ }^{44}$ examined the motion of pendant hydrogens in detail. Their analysis revealed a crossover from Gaussian to nonGaussian behavior with increasing q. In the q-range bel ow $q_{\max }$, where $q_{\max }$ is the $q$ value corresponding to the first maximum of the static structure factor, $G_{s}(q, t)$ and I (q,t) were found to display Gaussian behavior, with $\tau_{K w w}(q)$ scaling ${ }^{45}$ as $q^{-2 / \beta}$. In the q-range above $q_{\max }$, strong deviations from Gaussian behavior were observed, with $\tau_{\mathrm{kww}}(\mathrm{q})$ scaling as $\mathrm{q}^{-2}$. In Figure 21 we see that our results for the pendant hydrogens of aPP at $260 \mathrm{~K}$ display a crossover very similar to that reported by Colmenero et al. Two linear regimes are discerned in the log-log plot of $\tau_{\mathrm{Kww}}(\mathrm{q})$ over the displayed q-range, with slopes of -3.9 and -2 for small $q$ and large $q$, respectively. The two regimes cross at a q value very close to $q_{\max }=1.1 \AA^{-1}$, observed for aPP. ${ }^{3}$ I nterestingly, a sharp crossover is not seen for the pendant hydrogens at $350 \mathrm{~K}$ and at higher temperatures (not shown in Figure 21), nor for the $\tau_{\mathrm{K} w w}(\mathrm{q})$ values determined from analysis of the motion of all hydrogens (pendant and methyl) in the system (Figure 20).

Perhaps the most direct comparison of simulation predictions against experimental data is performed at the level of the incoherent dynamic structure factor $\mathrm{S}(\mathrm{q}, \omega)$, which is the experimental observable in QENS. Such comparisons against the ISIS and NEAT data are shown in Figures 22 and 23, respectively. The experimental data, originally obtained over asymmetric energy windows, have been symmetrized in the plots for easier comparison. The instrumental resolution function is shown as a broken line in all cases. The incoherent dynamic structure factor $S(q, \omega)$ was calculated by Fourier transforming the piecewise analytical representation of $I(q, t)$ after multiplication by the resolution function $\mathrm{R}(\mathrm{t})$ in the time domain. ${ }^{15} \mathrm{~F}$ igure 22 compares the predicted $S(q, \omega)$ at $450 \mathrm{~K}$ against the ISIS measure ments at $440 \mathrm{~K}$ for a q value of $1.69 \AA^{-1}$. The same comparison in the time domain has been presented as part of Figure 14. Agreement is satisfactory for energy transfers bel ow $0.2 \mathrm{meV}$ (time scales Ionger than $20 \mathrm{ps)}$ ), with the simulated spectrum being somewhat broader. 


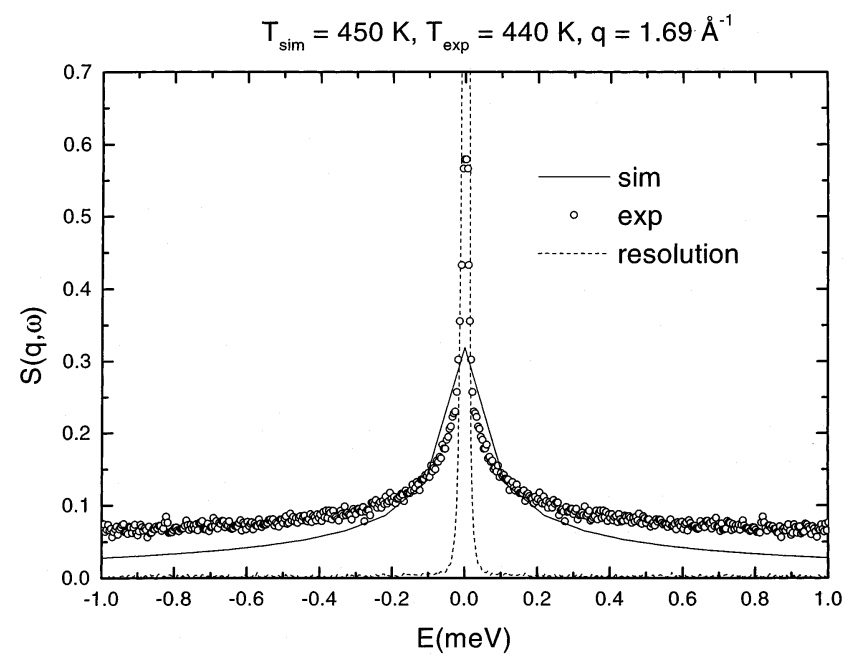

Figure 22. Predicted incoherent dynamic structure factor $\mathrm{S}(\mathrm{q}, \omega)$ at $450 \mathrm{~K}$ being compared to the experimental spectrum obtained at ISIS (points) at $440 \mathrm{~K}$ for $\mathrm{q}=1.69 \AA^{-1}$. The instrumental resolution function is shown as a broken line.
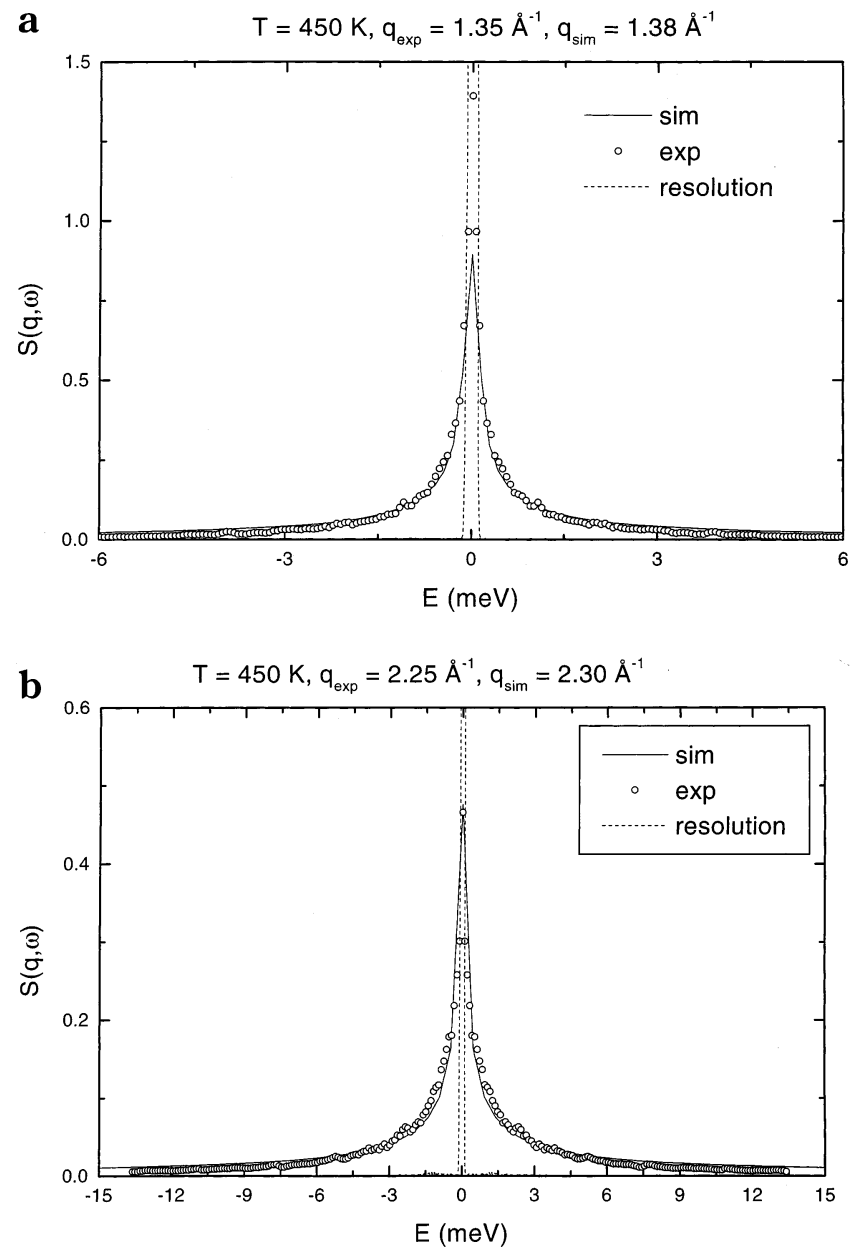

Figure 23. Predicted incoherent dynamic structure factors $\mathrm{S}(\mathrm{q}, \omega)$ at $450 \mathrm{~K}$ being compared to the experimental spectra obtained at NEAT (points). The instrumental resolution function is shown as a broken line. (a) $q=1.35 \AA^{-1}$ in the experiment and $q=1.38 \AA^{-1}$ in the simulation; (b) $q=2.25$ $\AA^{-1}$ in the experiment, $q=2.30 \AA^{-1}$ in the simulation.

The wings of the simulated $S(q, \omega)$ are narrower than in the measurement, reflecting that the simulated I $(q, t)$ falls significantly faster than its experimental counterpart in the range $4-20 \mathrm{ps}$ (see Figure 14). Parts a and $\mathrm{b}$ of Figure 23 compare the predicted $\mathrm{S}(\mathrm{q}, \omega)$ at $450 \mathrm{~K}$

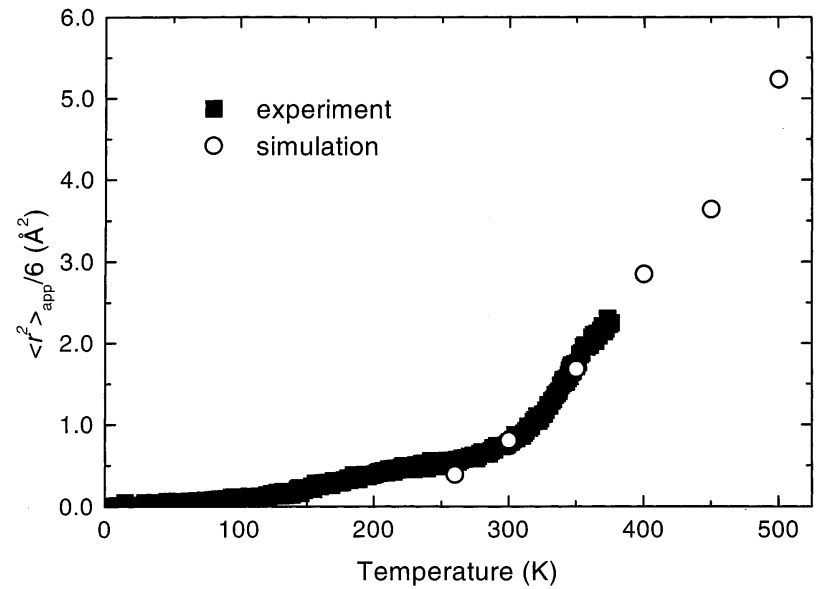

Figure 24. Apparent mean square displacements of hydrogen atoms, as extracted from the q-dependence of the elastic incoherent stucture factor of the fixed-window scan measurements, being compared to the mean square displacements of hydrogen atoms in the simulation, for the same time of observation. Results are shown for the entire temperature ranges examined in the simulation and in the experiment.

against NEAT measurements at $\mathrm{q}=1.4 \AA^{-1}$ and at $\mathrm{q}=$ $2.3 \AA^{-1}$, respectively. Agreement between computed and experimental $\mathbf{S}(q, \omega)$ curves in Figure 23 is very good, indicating that the simulation captures the time scales of segmental motion quite well over a variety of length scales. In particular, the wings of $\mathbf{S}(q, \omega)$ are very well predicted by the simulation. Over the energy window of the NEAT measurement, the shape of these wings is strongly affected by the fast initial exponential decay of I $(q, t)$ (see Figure 13); clearly, our simulation picture of this decay provides a good representation of reality.

A feature not reproduced by the simulation is the sharp "elastic" peak seen at $\omega \approx 0$ in Figures 22 and 23a. This peak indicates the presence, in the experimental samples, of slow motions (in comparison to the resolution of the ISIS measurements), which are absent from the simulation. The same sl ow motions are evident in the leveling off of the experimental I $(q, t)$ curves of Figure 14 at nonzero values for long times. These slow motions may be due to deviations of the stereochemical configuration in experimental samples from the welldefined Bernoullian distribution assumed in the modeling. Long syndiotactic sequences encourage trans conformational states ${ }^{20}$ and could conceivably lead to the formation of more slowly relaxing aggregates. To our knowledge, this is the first time that a pronounced elastic peak has been observed in a polymer melt at high temperature, i.e., ca. $200 \mathrm{~K}$ above the glass temperature. The precise origin of this el astic component is not clear at this time.

Figure 24 displays the apparent mean square displacement of hydrogen atoms, $\left\langle r^{2}\right\rangle_{a p p}$, calculated via eq 10 from the fixed window scan measurements in the temperature range $2-400 \mathrm{~K}$. As stated in section 4 , the resolution of the measurement was $1 \mu \mathrm{eV}$, corresponding to a time scale of $4.1 \mathrm{~ns}$. Plotted along with the experimental data are values of the actual mean squared displacement of hydrogen atoms, $\left\langle\mathrm{r}^{2}(\mathrm{t})\right\rangle$, at a time $\mathrm{t}=$ $4.1 \mathrm{~ns}$, as obtained from our simulations with model II in the temperature range $260-500 \mathrm{~K}$. Interestingly, the two sets of points are in good agreement over the temperature region where they overlap. 


\section{Conclusions}

The dynamics of atactic polypropylene has been expl ored with quasielastic neutron scattering measurements using two samples and three different instruments, covering a temperature range of $4-460 \mathrm{~K}$, a momentum transfer range of $q=0.2-2.25 \AA^{-1}$, and an energy transfer range of -2 to $10 \mathrm{meV}$. In parallel, molecular simulations of the same polymer have been conducted in the temperature range $260-600 \mathrm{~K}$ in order to calculate the observables of QENS over the same momentum and energy transfer ranges and to elucidate the motional processes that shape these observables.

The simulations have been carried out using two different molecular models: a fully explicit (all-atom) model and a coarser model that employs united-atom methyl groups and thereby can access long times at lower computational cost. Using MD simulations of the fully explicit model, we have analyzed the detailed mechanism and time scales of torsional librations and conformational jumps of methyl groups around their stem. Methyl groups were found to move essentially independently of each other, their conformational transitions being subject to a Gaussian distribution of activation energies with mean $\mathrm{E}_{\mathrm{A}}=14.7-15.3 \mathrm{~kJ} / \mathrm{mol}$ and standard deviation $\sigma=3 \mathrm{~kJ} / \mathrm{mol}$. The latter values compare well with the values $\mathrm{E}_{\mathrm{A}}=14.3 \mathrm{~kJ} / \mathrm{mol}$ and $\sigma$ $=3.3 \mathrm{~kJ} / \mathrm{mol}$ extracted from an analysis of our lowtemperature fixed window scan measurements.

A simple strategy is proposed for introducing the motion of methyl hydrogens around the methyl stems a posteriori in the model with united-atom methyls in a manner consistent with the fully atomistic MD simulations and with experiment. Incoherent intermediate scattering function $\mathrm{I}(\mathrm{q}, \mathrm{t})$ obtained in this way can be described in terms of four regimes: The initial, shorttime regime extends to ca. 1 ps at all temperatures. In this regime, the decay of $I(q, t)$ with time is close to exponential; it is caused by very fast (period $0.14 \mathrm{ps)}$ librations of the methyls around their stems, bond angle bending vibrations, and torsional oscillations of the backbone bonds. In the second regime, torsional jumps of methyl groups come into play, the time between successive jumps ranging from ca. $1.2 \mathrm{ps}$ at $600 \mathrm{~K}$ to ca. $52 \mathrm{ps}$ at $260 \mathrm{~K}$. In a third, longer time regime, correlated torsional transitions of the backbone bonds appear and become widespread at high temperatures, leading to the principal $\alpha$-relaxation of the polymer. There is no sharp distinction between the second and third regimes. Within the accuracy of the simulations, the time decay of I $(q, t)$ in these two regimes is very well described by a stretched exponential function, the stretching exponent being around 0.5 at $600 \mathrm{~K}$ and decreasing with decreasing temperature. At the higher temperatures and times beyond 100 ps, one can detect a fourth regime wherein diffusional motions of the (relatively short) chains constituting the melt affect the low-q dynamics significantly and the time decay of I (q,t) becomes again closer to exponential.

Computed I (q,t) curves from the simulation are in reasonable agreement with the corresponding curves obtained by inverse F ourier transformation of the QE NS data, with the exception of an elastic contribution seen in the experiments at long times. Correlation times extracted from the simulation at $\mathrm{q}=1 \AA^{-1}$ are consistent with the corresponding times extracted from both incoherent and coherent dynamic neutron scattering measurements in terms of their magnitude and tem- perature dependence; they are also consistent with NMR correlation times and estimates of the latter obtained from past simulation work. The q-dependence of KWW times extracted from fitting the long-time part of $I(q, t)$ (beyond the fast initial exponential decay) is consistent with experiment and past simulations on other polymers.

Incoherent dynamic structure factors $\mathrm{S}(\mathrm{q}, \omega)$ predicted by Fourier transformation of the simulation results, with due consideration of the instrumental resolution function, are in reasonable agreement with measured spectra. Furthermore, atomic mean square displacements from the simulation are in good agreement with estimates based on the q-dependence of the elastic incoherent structure factor from the fixed-window scan measurements at various temperatures.

Acknowledgment. Support from Directorate General 12 of the European Commission in the context of the TMR Research Training Network "New Routes to Understanding Polymers Using Experiments and Realistic Modeling" (NEWRUP), Contract NoERB-FMRXCT98-0176, is gratefully acknowledged. We thank Manolis Doxastakis for his guidance in the calculation of QENS observables and Professor Françoise Lauprêtre for stimulating discussions. We thank Professor Mark Ediger for making ref 22 available to us prior to its publication. We also thank Professor J uan Colmenero for sharing ref 44 with us before its publication.

\section{References and Notes}

(1) McCrum, N. G.; Read, B. E.; Williams, G. Anelastic and Dielectric Effects in Polymer Solids; Wiley: New York, 1967.

(2) Frick, B.; Richter, D. Science 1995, 267, 1939.

(3) Antoniadis, S. J .; Samara, C. T.; Theodorou, D. N. Macromolecules 1998, 31, 7944.

(4) Smith, G. D.; Paul, W.; Yoon, D. W.; Zirkel, A.; Hendricks, J .; Richter, D.; Schober, H. J . Chem. Phys. 1997, 107, 4751.

(5) Paul, W.; Smith, G. D.; Yoon, D. Y.; Farago, B.; Rathgeber, S.; Zirkel, A.; Willner, L.; Richter, D. Phys. Rev. Lett. 1998, 80, 2346.

(6) Moe, N. E.; Ediger, M. D. Phys. Rev. E 1999, 59, 623.

(7) Smith, G. D.; Paul, W.; Monkenbusch, M.; Richter, D. Chem. Phys. 2000, 261, 61.

(8) Alvarez, F.; Arbe, A.; Colmenero, J .J . Chem. Phys. 2000, 261, 47.

(9) Alvarez, F.; Arbe, A.; Colmenero, J .; Nicholson, T. M.; Davies, G. R. Macromol ecules 2000, 33, 8077.

(10) Saelee, C.; Nicholson, T. M.; Davies, G. R. Macromolecules 2000, 33, 2258.

(11) Zorn, R.; Richter, D.; Farago, B.; Frick, B.; Kremer, F.; Kirst, U.; Fetters, L. J. Physica B 1992, $180 \&$ 181, 534.

(12) Mos, B.; Verkerk, P.; Pouget, S.; van Zon, A.; Bel, G.-J .; de Leew, S. W.; Eisenbach, C. D. J . Chem. Phys. 2000, 113, 4.

(13) de Leeuw, S. W.; van Zon, A.; Bel, G. J . Electrochem. Acta 2001, 46, 1419.

(14) Borodin, O.; Smith, G. D. Macromolecules 2000, 33, 2273.

(15) Doxastakis, M.; Kitsiou, M.; Fytas, G.; Theodorou, D. N.; Hadjichristidis, N.; Meier, G.; Frick, B.J . Chem. Phys. 2000, 112,8687

(16) Smith, G. D.; Yoon, D. Y.; Wade, C. G.; O'Leary, D.; Chen, A.; J affe, R. L. J . Chem. Phys. 1997, 106, 3798.

(17) Smith, G. D.; Paul, W.; Monkenbusch, M.; Willner, L.; Richter, D.; Qiu, X. H.; Ediger, M. D. Macromol ecules 1999, 32, 8857.

(18) Smith, G. D.; Borodin, O.; Bedrov, D.; Paul, W.; Qiu, X.; Ediger, M. D. Macromol ecules 2001, 34, 5192.

(19) Destrée, M.; Lauprêtre, F.; Lyulin, A.; Ryckaert, J .-P. J. Chem. Phys. 2000, 112, 9632.

(20) Antoniadis, S. J .; Samara, C. T.; Theodorou, D. N. Macromolecules 1999, 32, 8635.

(21) Moe, N. E.; Qiu, X. H.; Ediger, M. D. Macromolecules 2000, 33, 2145; Qiu, X. H.; Moe, N. E.; Ediger, M. D.; Fetters, L. J . J. Chem. Phys. 2000, 113, 2918.

(22) Lippow, S. M.; Qiu, X. H.; Ediger, M. D. J . Chem. Phys. 2001 $115,4961$. 
(23) Ryckaert, J .-P.; Arialdi, G.; Melchionna, S. Mol. Phys. 2001, 99, 155.

(24) Melchionna, S.; Cozzini, S. DLPROTEIN 1.2 User Manual, ftp://ftp.dl.ac.uk/ccp5/DL_POLY/DL_POLY_2.0/documents/ DLPROTEIN 1.0.ps.

(25) Suter, U. W. Macromolecules 1981, 14, 523; Suter, U. W.; Neuenschwander, P. Macromol ecules 1980, 14, 528.

(26) Arrighi, V.; Ferguson, R.; Lechner, L. E.; Telling, M.; Triolo, A. Physica B 2001, 301, 35.

(27) Nicholson, T. M.; Davies, G. R. Macromolecules 1997, 30 , 5501.

(28) Arrighi, V.; Higgins, J. S.; Mani, S.; Stoeber, L. Physica B 1999, 226, 1.

(29) Mukhopadhyay, R.; Alegría, A.; Colmenero, J .; Frick, B. Macromolecules 1998, 31, 3985.

(30) Kramers, H. A. Physica 1940, 7, 284

(31) Weiner, J.H. Statistical Mechanics of Elasticity; Wiley: New York, 1983.

(32) Birr, M.; Alefeld, B.; Heidemann, A. Nucl. Instrum. Methods 1971, 95, 5435.

(33) Frick, B.; Fetters, L. J . Macromolecules 1994, 27, 974.

(34) Howells, W. S. A Fast Fourier Transform Program for the Deconvolution of the IN-10 Data; RL-81-039, Rutherford Appleton Laboratory, 1981.
(35) Arbe, A.; Colmenero, J .; Frick, B.; Monkenbusch, M.; Richter, D. Macromolecules 1998, 31, 4926.

(36) Zorn, R.; Frick, B.; Fetters, L. J . J . Chem. Phys. 2002, 116, 845.

(37) Lechner, R. E.; Melzer, R.; Fitter, J. Physica B 2001, 226, 86.

(38) Provencher, S. Comput. Phys. Commun. 1982, 27, 229.

(39) Chahid, A.; Alegría, A.; Colmenero, J . Macromol ecules 1994 $27,3282$.

(40) Horbach, J .; Kob, W. Phys. Rev. E 2001, 64, 041503.

(41) Dekmezian, A.; Axelson, D.; Dechter, J . J .; Borah, B.; Mandelkern, L. J . Polym. Sci: Polym. Phys. Ed. 1985, 23, 367.

(42) Colmenero, J .; Alegría, A.; Alberdi, J . M.; Alvarez, F., et al. Phys. Rev. B 1991, 44, 7321.

(43) Arrighi, V.; Pappas, C.; Triolo, A.; Pouget, S. Physica B 2001, $301,157$.

(44) Colmenero, J .; Alvarez, F.; Arbe, A. Phys. Rev. E 2002, 65, 041804.

(45) Colmenero, J .; Alegría, A.; Arbe, A.; Frick, B. Phys. Rev. Lett. 1992, 69, 478.

MA011807U 